\title{
The mitochondria-associated membrane protein PACS2 promotes mitophagy and energy metabolism to maintain right cardiac function in hypobaric hypoxia exposure
}

Jie Yang

Third Military Medical University

Mengjia Sun

Army Medical University

Renzheng Chen

Army Medical University

Chuan Liu

Third Military Medical University

Jihang Zhang

Xubin Gao

Army Medical University

Yuanqi Yang

Third Military Medical University

\section{Xiaohan Ding}

Army Medical University

\section{Ran Cheng}

Army Medical University

Chunyan He

Army Medical University

Shizhu Bian

Third Military Medical University

Zhengyuan Yuanfang

Third Military Medical University

Xiaowei Ye

Army Medical University

Boji Wu

Army Medical University

Lan Huang ( $\nabla$ huanglan260@126.com )

Army Medical University https://orcid.org/0000-0001-6200-2309 


\section{Article}

Keywords: PACS2, cardiomyocyte injury, right cardiac function, hypobaric hypoxia, mitophagy, mitochondria-associated membrane

Posted Date: January 17th, 2022

DOI: https://doi.org/10.21203/rs.3.rs-1249680/v1

License: (c) (i) This work is licensed under a Creative Commons Attribution 4.0 International License.

Read Full License 


\section{Abstract}

Hypobaric hypoxia $(\mathrm{HH})$ is the primary challenge at high altitude. Prolonged $\mathrm{HH}$ exposure impairs right cardiac function. Mitochondria-associated membrane (MAM) plays a principal role in regulating mitochondrial function under hypoxic conditions, but the mechanism remains poorly understood. In this study, proteomics analysis identified that PACS2, a key protein in MAM, and mitophagy were downregulated in $\mathrm{HH}$ conditions. Metabolomics analysis indicated suppression of aerobic oxidation of glucose and fatty acids. Cardiomyocyte Pacs2 deficiency disrupted MAM formation and endoplasmic reticulum (ER)-mitochondria calcium flux further inhibiting mitophagy and mitochondrial energy metabolism during $\mathrm{HH}$ exposure. Overexpression of Pacs2 reversed these effects. Cardiac-specific knockout of Pacs2 exacerbated mitophagy inhibition, cardiomyocyte injury and right cardiac dysfunction induced by $\mathrm{HH}$. Knock-in of Pacs2 recovered $\mathrm{HH}$-induced RV structural and functional impairment. Thus, PACS2 is essential for protecting cardiomyocytes through mechanisms of ER-mitochondria calcium flux, mitophagy, and mitochondrial energy metabolism, thereby maintaining right cardiac function at high altitude.

\section{Introduction}

High-altitude areas cover much of the total geographical area worldwide, and human exposure to high altitudes is increasing for various reasons. Hypobaric hypoxia $(\mathrm{HH})$ caused by increasing altitude is the main physiological challenge in such conditions and has long been recognised as a cause of cardiac stress. Acute exposure to high altitudes induces an increase in the right ventricular (RV) afterload, leading to alteration of the RV filling patterns ${ }^{1}$. Prolonged exposure to high altitude conditions further results in chronic remodelling of the cardiac structure and function, ultimately leading to right heart failure ${ }^{2,3}$. Among these cardiac adaptive and/or pathological alterations, cardiomyocyte responses, particularly in intracellular homeostasis maintenance during hypoxia, are the critical molecular basis that determine the adaptive cardiac outcomes ${ }^{4}$.

Cardiomyocytes consume majority of oxygen in the mitochondria as an electron donor for oxidative phosphorylation (OXPHOS) ${ }^{5}$. Thus, mitochondria are highly sensitive to decreases in oxygen levels in cardiomyocytes. Hypoxia increases oxidative stress and mitochondrial DNA mutation and causes mitochondrial dysfunction ${ }^{6}$. Hypoxia also suppresses mitochondrial OXPHOS and leads to the accumulation of aerobic metabolic substrates and anaerobic metabolites, which result in cardiomyocyte injury and cardiac dysfunction ${ }^{7}$. These damaged mitochondria and excessive metabolic substrates could be removed by mitophagy 8,9 . However, mitophagy is suppressed in some cardiac diseases, such as diabetic cardiomyopathy and ischemic cardiomyopathy ${ }^{10}$, which leads to mitochondrial dyshomeostasis and cardiac dysfunction. Therefore, an appropriate level of mitophagy serves as a protective mechanism to maintain the mitochondrial function in response to cardiac stress ${ }^{11}$. In recent years, several mitochondrial membrane receptors containing the LC3-interacting region (LIR) motif have been found to mediate mitophagosome formation under acute hypoxic conditions ${ }^{8,12}$. However, there is a paucity of 
information regarding cardiomyocyte mitophagy during chronic $\mathrm{HH}$ exposure and the precise mechanism has not been fully elucidated.

Recent studies indicated that cardiomyocyte mitochondrial function is regulated by the mitochondriaassociated membrane (MAM) structure, tethering two organelles by proteins located on opposing membranes ${ }^{13,14}$. Stable contact between the endoplasmic reticulum (ER) and the mitochondria integrates the two organelles' functions to regulate autophagy ${ }^{15}$, calcium signalling ${ }^{16}$, and others. Phosphofurin acidic cluster sorting protein 2 (PACS2) is a key physical linkage protein on the MAM, connecting the ER with the mitochondria and maintaining MAM stability. The physiological function of the MAM, particularly the calcium flux from the ER to the mitochondria and the transport of membrane phospholipids, relies on PACS $2{ }^{17}$. Previous studies have reported the important role of PACS2 in the development and progression of several tumours ${ }^{18-20}$. Tumour cells survive in a hypoxic microenvironment because of their infinite proliferation. Therefore, PACS2 may be an effector of hypoxia and may also be involved in the cell responses at high-altitude conditions, characterised by $\mathrm{HH}$.

In this study, we established HH conditions to closely simulate high-altitude exposure and focused on the PACS2-mediated mitophagy and mitochondrial energy metabolism, regarding the calcium flux across the MAM as the core mechanism. This study provided insight into the cardiomyocyte response to $\mathrm{HH}$ conditions. We also interpreted the mechanism underlying high-altitude-induced right cardiac dysfunction.

\section{Results}

Proteomics revealed down-regulated PACS2, mitophagy, and mitochondrial energy metabolism in the right myocardium in response to hypobaric hypoxia. $\mathrm{C} 57 \mathrm{BL} / 6 \mathrm{~J}$ mice were assigned to a $\mathrm{HH}$ chamber to simulate high-altitude conditions for 6 weeks (Fig. 1a). The right cardiac function of the mice was detected by echocardiography and RHC. As depicted in Figure 2, the mice subjected to $\mathrm{HH}$ conditions showed a markedly lower RV FAC (Fig. 2a, d) and VTI (Fig. 2h), concurrent with an increase in the mPAP (Fig. 2c, f), Tei index (Fig. 2b, e), and max dP/dt (Fig. 2g), indicating impaired right cardiac function. Additionally, increased heart mass (Fig. 2i, j) and Fulton index (Fig. 2k) were observed after the 6-week HH exposure. The abovementioned results indicated a stable RV dysfunction generated after 6-week chronic $\mathrm{HH}$ exposure.

Next, we obtained the right myocardium and performed proteomics and metabolomics analyses, accordingly. The different candidates were defined using a criterion of $\geq 1.2$-fold change and a significant difference between the groups. In the proteomics analysis, we identified 217 down-regulated proteins and 82 up-regulated proteins in the right myocardium of $\mathrm{HH}$-exposed mice compared with the respective levels in the NN counterparts (Fig. 1b, Supplemental Table 1). In the metabolomics analysis, we identified that 53 endogenous metabolites increased and 22 decreased (Supplemental Table 2). Hierarchical clustering analysis of metabolomics indicated markedly altered cardiac metabolic pathways under $\mathrm{HH}$ exposure (Fig. 1c). Among the differential expressed proteins, we identified PACS2 as being 
significantly down-regulated by 3.16 folds (Fig. 1d). In addition to PACS2, MAP1LC3A, MAP1LC3B, autophagy-related 16-like 1, and sequestosome 1 (SQSTM1/p62) were remarkably down-regulated, which were involved in phagophore formation and mitophagy induction (Fig. 1e). To gain insight into the possible biological impact of $\mathrm{HH}$ exposure, we subjected proteins that were down- or upregulated to KEGG and GO pathway enrichment analysis. The glycolysis, HIF-1 signaling pathway, and focal adhesion were up-regulated during $\mathrm{HH}$, while the OXPHOS, citrate cycle, fatty acid beta-oxidation, and mitophagy were down-regulated (Fig. $1 \mathrm{f}$ and $1 \mathrm{~g}$ ). The abovementioned results indicated that both mitophagy and mitochondrial energy metabolism were impaired in the right myocardium of the mice due to $\mathrm{HH}$ exposure.

Cardiac Pacs2 ablation exacerbated the right cardiac dysfunction and structure impairment induced by hypobaric hypoxia exposure. To determine the role of PACS2 in maintaining right cardiac function and structure, we generated cardiomyocyte-specific Pacs2 $2^{-/-}\left(\mathrm{Pacs}^{\text {flox/flox}} / \mathrm{Cre}^{\mathrm{aMHC+/-}}\right)$ mice. The Pacs2 ${ }^{-/-}$ mice and their littermate controls ( $\mathrm{Pacs}^{\mathrm{wt} /+} / \mathrm{Cre}^{\mathrm{aMHC+/-}}$ ) underwent echocardiography and $\mathrm{RHC}$ evaluation after a 6-week $\mathrm{HH}$ exposure. During exposure to $\mathrm{HH}, \mathrm{Pacs}^{-/-}$mice exhibited a significantly lower RV FAC (Fig. 2a, d) and higher Tei index (Fig. 2b, e) than their littermate controls. Interestingly, we observed that other parameters reflecting the afterload, including the mPAP (Fig. 2c, f), max dP/dt (Fig. 2g), and RV VTI (Fig. 2h), were not further aggravated in Pacs2 ${ }^{-/-}$mice during $\mathrm{HH}$ exposure. The heart weight was not further changed in $\mathrm{Pacs}^{-/-}$mice (Fig. 2j); however, cardiomyocyte-specific knocking out of PACS2 resulted in right ventricular hypertrophy based on a significant increase in the Fulton Index (Fig. 2k). HE staining further revealed increased RV chamber thickness, decreased RV chamber size, and disordered arrangement of RV myocardium caused by cardiac Pacs2 ablation (Fig. 3a). Masson's trichrome staining demonstrated significant collagen deposition in the right myocardial interstitial space after HH exposure (Fig. 3b, c). The mean cross-sectional area (CSA) of the $\mathrm{RV}$ cardiomyocytes in the HH group was significantly larger than that in the NN group (Fig. 3d, e). Such substantial right cardiac remodelling caused by $\mathrm{HH}$ was significantly more serious in the hearts of $\mathrm{Pacs}^{-/-}$mice. The cardiomyocyte injury was also evidenced by plasma markers (BNP, Tnl, and CK-MB), which were much higher in $\mathrm{Pacs}^{-/-}$mice than in the remaining two groups (Fig. $3 f-h$ ). Our results indicated that $\mathrm{HH}$-induced right cardiac impairment phenotype became more noticeable following Pacs 2 ablation.

To verify the separate role of Pacs2 ablation, we evaluated the right cardiac function under NN conditions. Compared to littermate controls, Pacs $^{-/-}$mice showed a normal mPAP (Supplemental Fig. 1c, 1f-h), which was accompanied by impaired right cardiac function, as revealed by lower RV FAC (Supplemental Fig. 1a, d) and an increased Tei index (Supplemental Fig. 1b, e). Pacs2 ${ }^{-/}{ }^{-}$mice in NN exhibited significantly increased myocardial disorder and fibrosis (Supplemental Fig. 2a-c). Additionally, cardiomyocyte injury was also evident in NN conditions in the presence of Pacs2 $^{-/-}$(Supplemental Fig. 2d-h). In general, Pacs2 ablation exacerbated the cardiomyocyte injury and right cardiac dysfunction; however, it did not act on the RV afterload during $\mathrm{HH}$ exposure. 
Cardiac Pacs2 ablation exacerbated MAM disruption and mitophagy reduction induced by hypobaric hypoxia. To determine how PACS2 responds to $\mathrm{HH}$, we compared the subcellular localization of MAMassociated proteins in isolated right myocardium under $\mathrm{HH}$ or NN conditions. As shown in Fig. 4a, different fractions were identified with the following organelle markers: FACL4, VDAC1, MFN2, FIS1, TOMM20, CNX, and DRP1. The level of the PACS2 in MAM significantly decreased in the HH group compared with its levels in the NN counterparts, although a small amount of PACS2 can also be found in the cytosol. However, the levels of other MAM-related proteins were not noticeably altered in MAM fractions isolated from the $\mathrm{HH}$ group. To assess whether PACS2 affects MAM integrity, we examined the ER-mitochondrial contacts in Pacs2 ${ }^{-/-}$myocardium. As illustrated by the TEM images (Fig. 4b, c), the proportion of ER in close contact with mitochondria relative to the total ER content was lower in the $\mathrm{HH}$ group than in the NN group and further decreased in the Pacs2 ${ }^{-/-}$mice. Consistent with the TEM images, immunofluorescence analysis clearly showed a lower level of co-localization of the ER with mitochondria in $\operatorname{Pacs}^{-/-}$mice than in the remaining groups (Fig. 4d, e).

Next, we determined whether PACS2 alteration affected mitophagy. Impaired mitophagy induced by HH exposure was confirmed by decreased mitophagy markers in the right myocardium. The blotting results indicated that the level of MAP1LC3B-II was lower in Pacs2 ${ }^{-/-}$mice than in the controls during $\mathrm{HH}$ conditions (Fig. 4f, g). Moreover, immunostaining analysis revealed that Pacs2 deletion further reduced the co-localization of MAP1LC3B puncta and mitochondria induced by HH exposure (Fig. 4h, i). Taken together, cardiac Pacs2 ablation exacerbated MAM disruption and mitophagy reduction induced by $\mathrm{HH}$.

Hypobaric hypoxia reduced MAM formation and mitophagy in vitro. To explore the impact of $\mathrm{HH}$ on the MAM structure and biological function, we also measured the levels of MAM-related proteins in $\mathrm{H} 9 \mathrm{C} 2$ cardiomyocytes exposed to simulated hypobaric hypoxia in vitro. As depicted in Fig. 5a, in line with the in vivo results above, the expression of PACS2 in the MAM from $\mathrm{HH}$-treated cells was lower than that in the MAM from NN-treated cells. Consistently, confocal imaging and Pearson's correlation coefficient analysis (Fig. 5b, c) showed a decreased association between the ER and mitochondria in simulated $\mathrm{HH}$-treated cardiomyocytes compared with that in the NN cells. TEM imaging showed swollen mitochondria and fewer mitochondria adjacent to the ER after $\mathrm{HH}$ exposure (Fig. $5 \mathrm{~d}$, e). These data suggested that $\mathrm{HH}$ decreases the MAM junction structure in cardiomyocytes.

Furthermore, we evaluated mitophagy levels in H9C2 cardiomyocytes. We found decreased MAP1LC3B-II transfer (Fig. 5f, g) and co-localization with mitochondria after $\mathrm{HH}$ exposure (Fig. 5h, i). To further verify the impaired mitophagy, we transfected pH-dependent mitochondrial protein Keima into the cardiomyocytes, which can shift from green to red as mitochondria are delivered to lysosomes. LSCM monitoring showed that $\mathrm{HH}$ induced a markedly decreased mitophagy index in the cardiomyocytes (Fig. 5j, k), indicating that $\mathrm{HH}$ decreased the number of mitophagosomes and impaired the mitophagy flux. The above results in the $\mathrm{H} 9 \mathrm{C} 2$ cell lines as well show that $\mathrm{HH}$ reduces MAM formation and mitophagy. 
Hypobaric hypoxia reduced ER-mitochondria calcium flux and mitochondrial oxidative phosphorylation in vitro. PACS2 was reported to maintain the junction of the MAM and regulate mitochondrial calcium flux ${ }^{21}$. In this study, we found that $\left[\mathrm{Ca}^{2+}\right]_{m}$ in the $\mathrm{H} 9 \mathrm{C} 2$ cardiomyocytes after $\mathrm{HH}$ treatment was markedly lower than that in the NN group (Supplemental Fig. 3a, b). To determine the origin of mitochondrial calcium, we incubated $\mathrm{H} 9 \mathrm{C} 2$ cardiomyocytes under $\mathrm{HH}$ or $\mathrm{NN}$ with a cytoplasmic $\mathrm{Ca}^{2+}$ chelator, BAPTAAM $(10 \mu \mathrm{M})$, in calcium-free HBSS for $10 \mathrm{~min}$. Mitochondrial calcium was labelled by a Rhod2-AM probe, and the cells were observed and measured under LSCM. TG- (an inhibitor of calcium pump, Fig. 6a, b) and ATP- (an indirect IP3R agonist, Fig. 6c, d) elicited ER-mitochondria calcium flux was lower in the HHtreated cells than in NN-treated cells. Inositol trisphosphate receptors (IP3R) are important ER calcium release channels ${ }^{22}$. Therefore, we added 2-APB, which blocked the release of calcium from IP3R. Comparable mitochondrial calcium to the $\mathrm{HH}$ exposure was observed when treated with 2-APB (Fig. 6e, f), suggesting that IP3R is required for maintaining the physiological mitochondrial calcium levels under NN conditions.

MAM formation and ER-mitochondrial calcium flux are essential for mitochondrial energy metabolism. Thus, we evaluated the effects of $\mathrm{HH}$ on cardiomyocyte mitochondrial energy metabolism using a Seahorse XF analyser to measure the mitochondrial respiration and glycolytic flux. We found that cardiomyocytes exhibited significant decreases in basal and maximal cellular oxygen consumption rate (OCR) in response to HH. ATP production and spare respiration capacity were also significantly lower after $\mathrm{HH}$ exposure (Fig. $6 \mathrm{~g}, \mathrm{~h}$ ). The extracellular acidification rate (ECAR) results indicated an increase in glycolysis and glycolytic capacity due to insufficient oxygen (Fig. 6i, j). In addition, OCR measurement with a medium containing BSA-conjugated palmitic acid significantly decreased after $\mathrm{HH}$ exposure (Fig. 6k, I). The abovementioned results demonstrate that after $\mathrm{HH}$ exposure, the cardiomyocytes displayed a metabolic reprogramming, which is represented by the restriction of FAO-related OXPHOS and a tendency to rely more on glycolysis than aerobic glycolysis for adapting to the HH condition. Considering that $\mathrm{HH}$ caused a decline in ER-mitochondria calcium flux, one mechanism that potentially accounts for the metabolic shift may be associated with the regulation of mitochondrial calcium.

\section{ER-mitochondria calcium flux is involved in PACS2-mediated mitophagy and mitochondrial energy} metabolism. We next determined the contributions of ER-mitochondria calcium in PACS2-induced mitophagy and mitochondrial energy metabolism. We obtained cardiomyocytes with stable overexpression of Pacs2 by lentiviral vector (LVV) infection (Fig. 7a). ER-mitochondria contacts increased in cells where Pacs2 was overexpressed (Fig. 7b, c). We found reversed $\left[\mathrm{Ca}^{2+}\right]_{\mathrm{m}}$ in LVVs-infected cardiomyocytes (Supplemental Fig. 3a, b). Similar results were observed in cultured cells with Pacs2 overexpression in the dynamics of mitochondrial calcium flux (Fig. 7d, e), indicating a source of calcium flux released from the ER. The regulation of calcium flux between the ER and mitochondria via IP3R is a major function of the $\mathrm{MAM}^{23}$. As depicted in Fig. $7 f$ and $7 \mathrm{~g}$, the restored calcium levels caused by the overexpression of Pacs 2 were partly blocked by 2-APB, suggesting that Pacs2 overexpression promoted ER calcium release in the MAM through IP3R. 
Additionally, higher MAP1LC3B-II levels were observed after LVVs-overexpression of Pacs2 (Fig. 7h), which could also be blocked by 2-APB (Fig. 7i), suggesting that the PACS2mediated ERmitochondria calcium flux was required for mitophagy. With the supplementation of PACS2, more MAP1LC3B puncta co-localized with mitochondria (Fig. 8a, b) and an increased mitophagy index were observed in $\mathrm{HH}$ conditions (Fig. 8c, d). These data demonstrated that PACS2 restored impaired mitophagy through enhanced ER-mitochondria calcium flux. To investigate whether ERmitochondria calcium flux was also involved in PACS2-mediated mitochondrial energy metabolism alteration, we compared the real-time changes in OCR and ECAR in the H9C2 cardiomyocytes with or without overexpression of PACS2 under $\mathrm{HH}$ treatment. With PACS2 supplementation, the declined basal respiration, ATP production, and the maximal respiration (Fig. 8e, f) as well as increased basal and maximal ECAR (Fig. 8g, h) induced by HH were significantly reversed. The recovery in mitochondrial respiration was also blocked by 2-APB treatment (Fig. 8e, g). To extend the hypothesis that PACS2 recovered OCR, which is supported by FAO, we further measured OCR in a medium containing palmitate-BSA as an exogenous FAO substrate. Notably, the cardiomyocytes showed a reversed OCR after the supplementation of PACS2 when compared with an empty vector control (Fig. 8i, j), which was significantly blocked on adding 2-APB. This indicates that PACS2 enabled HH-treated cardiomyocytes to switch from glycolysis to an increased reliance on FAO for ATP production, and this metabolic reprogramming at least partly depends on the calcium flux across MAM. Taken together, these data suggested that ER-mitochondria calcium flux was essential for PACS2mediated mitophagy maintenance and mitochondrial energy metabolism after $\mathrm{HH}$ exposure.

Cardiac Pacs2 knock-in alleviated HH-induced right cardiac dysfunction. LVVs-overexpression of Pacs2 significantly reversed MAM formation, mitophagy, and mitochondrial energy metabolism in cardiomyocytes in vitro. To verify the contributions of PACS2 in maintaining RV function during $\mathrm{HH}$ exposure in vivo, we generated cardiomyocyte-specific Pacs2 knock-in mice. Histological analysis of the hearts from the Pacs2 knock-in mice showed significantly decreased right cardiac hypertrophy (Fig. 8a), cardiac fibrosis area (Fig. 8b, c), and cardiomyocytes CSA (Fig. 8d, e) with HH exposure. In addition, the Pacs2 knock-in mice had lower plasma levels of BNP, Tnl, and CK-MB (Fig. $8 f-h$ ) than that of their littermate controls, indicating that PACS2 supplementation reduced the $\mathrm{HH}$-induced myocardial damage. Compared with their littermate controls, the Pacs2 knock-in mice exhibited a higher RV FAC (Fig. 9a, d) and lower Tei index (Fig. 9b, e). As expected, Pacs2 overexpression failed to alter mPAP (Fig. 9c, f), max dP/dt (Fig. 9g), and RV VTI (Fig. 9h) during HH exposure. These data suggested that conditional Pacs2 knock-in reduced cardiomyocyte injury and partially recovered RV cardiac function after $\mathrm{HH}$ exposure without significantly influencing the RV afterload.

\section{Discussion}

This is the first study to reveal the underlying mechanism of PACS2 in HH-mediated cardiomyocyte injury and right cardiac dysfunction. The core process was the down-regulated PACS2 localized in MAM after $\mathrm{HH}$ exposure. PACS2 reduction further suppressed MAM formation and resulted in decreased calcium flux from the ER to the mitochondria via the IP3R calcium channel. The reduction of mitochondrial calcium influx further inhibited mitophagy and mitochondrial energy metabolism, inducing cardiomyocyte injury 
and right cardiac dysfunction. Moreover, cardiomyocyte-specific knock-in of Pacs2 reversed right cardiac dysfunction and RV fibrosis. Of note, neither knockout nor knock-in of Pacs2 influenced the RV afterload, highlighting an independent role of PACS2-directed cardiomyocyte responses in maintaining right cardiac function. Thus, our results provided potential therapeutic targets for high-altitude induced right cardiac impairment.

Sufficient oxygen supply is the most essential condition for the survival and function of cardiomyocytes. Hypoxia induces pulmonary vasoconstriction and increases pulmonary vascular resistance. Prolonged hypoxia further results in right cardiac function impairment and even right heart failure ${ }^{24}$. Thus, in this study, we focused on the right rather than on the left cardiac function. Hypoxia is usually generated by normobaric hypoxia $(\mathrm{NH})$ or $\mathrm{HH}$ in the experiments. $\mathrm{NH}$ lowers the partial pressure of inspired oxygen $\left(\mathrm{PiO}_{2}\right)$ by reducing the fraction of inspired oxygen through the addition of exogenous nitrogen without altering the barometric pressure. Conversely, $\mathrm{HH}$ lowers the $\mathrm{PiO}_{2}$ by reducing the barometric pressure. Previous studies have suggested that $\mathrm{NH}$ and $\mathrm{HH}$ induced similar cardiac adaptations over a short duration, although lower $\mathrm{SpO}_{2}$ and worse right cardiac function emerged during long-term exposure ${ }^{25,26}$. Thus, more complicated mechanisms may exist in $\mathrm{HH}$ than in $\mathrm{NH}$, including intravascular bubble formation, increased alveolar deadspace, altered fluid permeability, and a mismatch in ventilation and perfusion ${ }^{25,27}$. Our study established a long-term $\mathrm{HH}$ exposure model to simulate real cardiac function alteration and cardiomyocyte response in high-altitude environments. Previous studies on cardiomyocyte injury caused by hypoxia mainly focused on the increase in oxygen free radicals and anaerobic metabolites, eventually leading to cardiomyocyte apoptosis, myocardial fibrosis, and irreversible cardiac remodelling ${ }^{28,29}$. Our study firstly indicated that both mitophagy and mitochondrial energy metabolism were involved in cardiomyocytes survival under $\mathrm{HH}$ conditions. Our results provided a novel mechanism that results in right cardiac dysfunction at high altitudes.

Previous studies reported that PACS2 is closely associated with the onset and progression of tumours, such as colorectal tumour and liver cancer ${ }^{18,30}$. Due to their infinite proliferation ability, tumour cells survive in relative hypoxic conditions. Therefore, it is essential to understand the biological function of PACS2 in regulating cell fate in response to hypoxic conditions. Accordingly, after $\mathrm{HH}$ exposure, PACS2 expression was found to be markedly reduced in the MAM, although with a moderate increase in PACS2 in the cytosol both in vivo and in vitro (Fig. 4a and Fig. 5a). This implied a dynamics translocation from the MAM to the cytosol, which may partly explain the decreased PACS2 in the MAM. The free form of PACS2 in the cytosol contributed to nuclear gene expression and membrane trafficking rather than calcium flux, thus exhibiting the suppression of downstream mitophagy and energy metabolism. Besides PACS2, recent studies have found that the FUN14 domain containing 1 (FUNDC1), a new protein in the MAM, is responsible for the release of calcium from the ER to the mitochondria and mitophagy induction in mouse cardiomyocytes ${ }^{31,32}$. Notably, our results revealed that PACS2 served as a new mitophagic regulator in the MAM and also modulated the release of calcium from ER to mitochondria in cardiomyocytes. However, the precise molecular mechanism of how PACS2 cooperates with FUNDC1 to regulate calcium flux, mitophagy, and cardiac function remains unknown. We considered that a few 
special proteins in the MAM may interact to form a protein complex or "protein machine" and get involved in the abovementioned process. PACS2 may be the key protein and serves as a scaffold to sponge other proteins.

Mitophagy is essential for mitochondrial homeostasis and quality control in cardiomyocytes ${ }^{33}$. During hypoxia, mitophagy is the sole mechanism by which cardiomyocytes eliminate superfluous or damaged mitochondria ${ }^{11}$. However, the mechanisms underlying mitophagy remain largely unknown. Previous studies on mitophagy have focused on several protein receptors, including BCL2 interacting protein 3 (BNIP3), BNIP3-like, and FUNDC1, on the mitochondrial membrane. Most of them have a classic LIR motif to directly bind MAP1LC3B for mitophagy activation ${ }^{34}$. In this study, we found a new protein in the MAM without this classic LIR motif, although it was closely associated with HH-mediated mitophagy. PACS2 did not directly link to autophagy-associated proteins, such as ATG5, ATG7, Beclin1, and MAP1LC3B; however, it acted as a calcium channel to promote calcium influx into the mitochondria ${ }^{21}$. Usually, intracellular calcium is considered an activator of autophagy 35,36 .

To date, the role of calcium signaling in autophagy regulation is highly controversial. Most studies considered that calcium works as an activator of autophagy because calcium mobilizing agents and calcium ionophores promote autophagy by elevating intracellular calcium concentration ${ }^{37}$. In this study, we detected that PACS2-mediated calcium influx was required for HH-induced mitophagy in cardiomyocytes, which further verified the impact of intracellular calcium on mitophagy regulation. However, the way in which mitochondrial calcium is involved in mitophagy activation requires further exploration. Mitochondrial calcium uptake occurs mostly through MAM, which closely contacts with the ER and renders a micro-domain with a sufficiently high calcium concentration ${ }^{38}$. A recent study reported that mitochondrial calcium influx inhibition decreased ATP production, enhanced mitophagy, and provided cardioprotection in cardiac failure ${ }^{39}$. Conversely, we found that $\mathrm{HH}$ decreased ER-mitochondria calcium flux and calcium-mediated mitophagy. Similar to our results, Böckler and Zou demonstrated that ERmitochondria contacts and calcium flux across the MAM were required for autophagic removal of mitochondria, since artificially tethering ER and mitochondria rescued mitophagy defects ${ }^{16,31}$. Besides mediating calcium flux, the ER-mitochondria encounter structure may also supply the growing phagophore with lipids synthesised in the ER, which then enclose the impaired mitochondria to form a mitophagosome. Hence, ER-mitochondria-mediated calcium flux is required for mitophagy induction.

In addition to the abovementioned role of calcium in mitophagy, mounting evidence suggests that calcium also dynamically regulates the aerobic energy metabolism by stimulating mitochondrial $\mathrm{OXPHOS}^{40,41}$. In highly energy-consuming tissues, such as the heart, OXPHOS in the mitochondria provides a major source of cellular ATP through the oxidation of substrates, including fatty acids, glucose, and ketones ${ }^{42}$. We found that to adapt to the $\mathrm{HH}$ condition, cardiomyocytes mainly rely on the glycolytic pathway rather than the OXPHOS pathway. As a critical signaling molecule in mitochondrial energy conversion, sufficient mitochondrial calcium concentration is required to activate the mitochondrial dehydrogenases including the pyruvate dehydrogenase complex (PDHC), NADH-isocitrate 
dehydrogenase $(I C D H)$ and a-ketoglutarate dehydrogenase $(a-K G D H)^{43,44}$. Other components within the energy-producing pathways besides NADH generation, such as downstream ATPase and the cytochrome chain, were also significantly stimulated by calcium ${ }^{45,46}$. Current studies supported the proposed physiological metabolic role for calcium entry into mitochondria matrix through mitochondrial calcium uniporter (MCU) complex ${ }^{47}$. Together with some recent reports, we indicated that the IP3R channels were also associated with alterations in mitochondrial calcium flux, especially in cardiomyocytes under $\mathrm{HH}$ exposure. IP3R-mediated calcium signaling required the quantification of PACS2 and the proximity of the ER and mitochondria. The supplement of PACS2 could improve mitochondrial respiration efficiency during $\mathrm{HH}$ exposure. Our previous randomized double-blinded clinical trial proposed that cardiac function could be recovered by optimizing myocardial energy metabolism ${ }^{48}$. Combined with those results, our present results provide a therapy for improving cardiac function at high altitude targeted on the energy metabolic reprogramming based on calcium flux across MAM in cardiomyocytes. Moreover, linking the reprogramming of energy metabolism induced by PACS2 supplement was associated with enhanced mitophagy. We considered that mitophagy may, at least partly, provide relatively efficient substrates such as fatty acids for maintaining energy demand. However, the exact role of calcium in cardiomyocyte energy metabolism reprogramming requires confirmation using accurate techniques such as isotope tracing analysis.

In conclusion, we described a novel cardiomyocyte injury mechanism during $\mathrm{HH}$ exposure to simulate high altitude. HH down-regulated the expression of PACS2 in MAM. Decreased PACS2 disrupted MAM formation and calcium transfer from the ER to the mitochondria, leading to mitophagy inhibition and mitochondrial energy metabolism impairment, which induced cardiomyocyte injury and right cardiac dysfunction during $\mathrm{HH}$ exposure. Our study identified a potential target for the prevention and treatment of cardiovascular diseases caused by high-altitude exposure.

\section{Methods}

Development of chronic hypobaric hypoxia-induced mouse model. All animal procedures were approved by the Experimental Animal Ethics Committee of the Army Medical University and conformed to the regulations of the Guide for the Care and Use of Laboratory Animals. Male C57BL/6J mice (6-8 weeks old) were housed in a temperature-controlled environment with a 12-hour light/dark cycle. The mice were subjected to $\mathrm{HH}$ condition (10.0\% oxygen content and $46.3 \mathrm{kPa}$ air pressure) in a chamber (AIPUINS XBS03, Hangzhou, China) or were housed in normobaric normoxia (NN, 20.9\% oxygen content and $101.3 \mathrm{kPa}$ air pressure) as controls for 6 weeks (Fig. 1a).

Blood preparation and enzyme-linked immunosorbent assay. Detection kits for mouse plasma B-type natriuretic peptide (BNP), troponin I (Tnl), and creatine kinase-MB (CK-MB) were purchased from Jiangsu Jingmei Biological Technology Co., Ltd. (Jiangsu, China). Approximately $1.5 \mathrm{~mL}$ of blood was drawn from each mouse and stored in procoagulant tubes. Plasma was separated by centrifugation $(3000 \times \mathrm{g}, 20$ min) after coagulation at room temperature for $10 \mathrm{~min}$. The plasma levels of BNP, Tnl, and CK-MB were measured using a commercially available BNP enzyme-linked immunosorbent assay (ELISA) kit (JM- 
02343M2, 210727B8), TnI ELISA kit (JM-02662M2, 210727I4), and CK-MB ELISA kit (JM-03084M2, 210727C6), respectively, following the manufacturer's instructions.

Hemodynamic monitoring. Right heart catheterization (RHC) was performed using a pressure detecting device (ADInstruments Mikro-Tip ${ }^{\circledR}$, MPVS Ultra RSBMIL002/M) after a 6-week HH or NN exposure. The mice were placed on a heated pad and anesthetized with $2 \%$ isoflurane. The right jugular vein was exposed, and a 1F needle (ADInstruments Mikro-Tip ${ }^{\circledR}$, SPR-1000) was slightly bent inwards to conduct the cannula containing the catheter into the jugular vein. The cannula was maneuvered to the right ventricle, with its tip pointing towards the heart until an RV pressure curve could be identified using LabChart 7 software. Next, the cannula tip was manipulated to the left and upward. The catheter was advanced into the main pulmonary artery, passing through the pulmonary valve. When the catheter enters the main pulmonary artery, the diastolic pressure rises on the monitor, and a pulmonary artery pressure curve appears. When the curve was constant, the related indices, such as the mean pulmonary artery pressure (mPAP), maximum positive time derivative of left ventricular pressure (max $\mathrm{dP} / \mathrm{dt}$ ), and RV velocity time integral (VTI) and electrocardiograms were measured.

Evaluation of right ventricular hypertrophy. After the hemodynamic measurement, the mice were sacrificed by cervical dislocation, and their hearts were removed quickly and weighed. The free wall of the right ventricle was dissected from the left ventricle and interstitial septum. Whole heart weight (normalized by body weight) and Fulton's index (right ventricle / [left ventricle + interstitial septum]) were used as indices of cardiac hypertrophy.

Histological analysis. The hearts from the mice exposed to $\mathrm{NN}$ and $\mathrm{HH}$ were excised, placed in $4 \%$ paraformaldehyde, dehydrated in graded concentrations of ethanol, immersed in xylene, and embedded in paraffin. Sections of $5-\mu \mathrm{m}$ thickness were cut on a microtome with a disposable blade, stained with hematoxylin-eosin and Masson's trichrome stain, and examined by light microscopy. The cardiomyocyte cross-sectional area (CSA) was analyzed by staining the heart sections with a wheat germ agglutininAlexa Fluor ${ }^{\circledR} 647$ conjugate (W32466, Invitrogen). Six mice from each group were included in the histological analysis. A minimum of five cross-sections of each heart were examined, and the measurements were averaged for statistical analysis. ImageJ software (RRID:SCR_003070) was used to quantify all the histological endpoints.

Generation of cardiomyocyte-specificPacs2knockout mice. Cardiomyocyte-specific Pacs 2 knockout $\left(\mathrm{Pacs}^{-/-}\right)$mice were generated on a C57BL/6J background by the CRISPR/Cas9 system at cyagen. The gRNA to mouse Pacs2 gene, the donor vector containing loxP sites, and Cas9 mRNA were co-injected into fertilized mouse eggs to generate targeted conditional knockout offspring. Pacs $2^{\text {flox/flox }}$ mice in which the Pacs2 gene was flanked by loxP sites within introns 1 and 3 (KO region: $\sim 1842$ bp) were crossed with amyosin heavy chain (a-MHC) promoter-Cre transgenic mice (Cyagen Biosciences) to obtain Pacs ${ }^{f l o x /+} / \mathrm{Cre}^{a \mathrm{MHC+/-}}$ mice. F0 founder animals were identified by PCR followed by sequence analysis, which were bred to wildtype mice to test germline transmission and F1 animal generation. F1 founders, 
including Cardiomyocyte-specific Pacs2 knockout (Pacs2 $2^{\text {flox/flox }} / \mathrm{Cre}^{a \mathrm{MHC}+/-}$ ) mice, were genotyped by tail genomic PCR.

Generation of cardiomyocyte-specificPacs2knock-in mice. The cardiomyocyte-specific Pacs2 knock-in in C57BL/6J mice was created using CRISPR/Cas-mediated genome engineering (Cyagen Biosciences). The Hipp11 locus is located within an intergenic region between the Eif4enif1 and Drg1 genes on mouse chromosome 11. The mouse Pacs2 gene (NCBI Reference Sequence: NM_001291444.1) is located on mouse chromosome 12. For the KI model, the "alphaMHC_long promoter-Kozak-Mouse Pacs2 CDS-rBG pA" cassette was inserted into Hipp11 locus ( 0.7 kb 5' of Eif4enif1 gene and $\sim 4.5 \mathrm{~kb} 3$ ' of the Drg1 gene). To engineer the targeting vector, homology arms were generated by PCR using BAC clone as the template. Cas9 and gRNA were co-injected into fertilized eggs with a targeting vector for mice production. The pups were genotyped by PCR followed by sequencing analysis.

Echocardiography. Cardiac geometry and function were examined using ultrasonography (GE Vivid 7 Dimension, $\mathrm{L} 15 / 6-\mathrm{MHz}$ transducer). The mice were anesthetized with $2 \%$ isoflurane while maintaining proper body temperature $\left(36-37^{\circ} \mathrm{C}\right)$ and heart rate $(450-550$ beats/ minute). The temporal frame rate in the echo mode was set to $60 \mathrm{~Hz}$. A 1.0-mm sampling gate was used to obtain the inflow and outflow velocities, and the maximal sweep speed was $200 \mathrm{~mm} / \mathrm{s}$. RV end-diastolic (ED) and end-systolic (ES) areas were measured using ImageJ from the apical or basal 4-chamber views at end-diastole or endsystole. The RV fractional area change (FAC) was calculated as follows: FAC = ([ED RV area - ES RV area] / ED RV area) $\times 100 \%$. For Tei index calculation, the tricuspid closure opening time (TCO) and ejection time (ET) were measured from tissue Doppler myocardial velocity images, as follows: Tei index = (TCO - ET) / ET. Data were collected from six mice per group and represented the average of minimum of five separate scans in a random blind fashion. To avoid bias, the researcher performed all echocardiography procedures blinded to the experimental treatments.

Cell culture and RNA transfection. Rat H9C2 cardiomyocytes (BFN60804388) were purchased from the Cell Bank of the Chinese Academy of Sciences (Shanghai, China). Cardiomyocytes were cultivated in Dulbecco's modified Eagle's medium (Sigma-Aldrich, Louis, MO, USA) and 10\% fetal bovine serum (Hy Clone Laboratories, PA, USA), and supplemented with $1 \%$ antibiotic-antimycotic $(1000 \mathrm{U} / \mathrm{mL}$ penicillin and $100 \mu \mathrm{g} / \mathrm{mL}$ streptomycin). $\mathrm{H} 9 \mathrm{C} 2$ cardiomyocytes in the NN group were incubated at $37^{\circ} \mathrm{C}$ with $5 \%$ $\mathrm{CO}_{2}$. $\mathrm{HH}$ conditions were achieved by using a $\mathrm{HH}$ chamber (Billups-Rothenberg) flushed with a preanalyzed gas mixture of $1 \% \mathrm{O}_{2}, 5 \% \mathrm{CO}_{2}$, and $94 \% \mathrm{~N}_{2}$. To maintain cardiomyocyte cultures, the medium was changed every 2 days. LVVs carrying Pacs2 RNA system were constructed by Gene Pharma Technology (Shanghai, China). The LVVs were added to the cells at a multiplicity of infection of 100 . The transfection medium was changed 2 days later, and the cells were continuously cultured in fresh medium. Real-time quantitative reverse transcription-PCR and western blotting were used to detect the efficiency of Pacs2 overexpression in cardiomyocytes.

Western blotting. Cardiomyocyte MAM fractions in vitro and MAM fractions of the hearts were isolated following a previously described protocol ${ }^{49}$. Western blotting was used to evaluate protein expression in 
different fractions. Briefly, the protein concentrations of different fractions after isolation were detected using the BCA assay (Beyotime Biotechnology, P0012). The same mass of total protein was separated by SDS-PAGE and transferred to polyvinylidene fluoride membranes (Millipore). The membranes were blocked with 5\% nonfat milk in Tris-buffered saline (Boster Biological Technology, AR0031) containing 0.5\% Tween-20 (Solarbio, T8220), and membrane-bound proteins were probed with primary antibodies purchased from Abcam against the following antigens: PACS2 (ab222316, Abcam), mitofusin 2 (MFN2; ab124773, Abcam), voltage-dependent anion-selective channel protein 1 (VDAC1; ab14734, Abcam), acylCoA synthetase 4 (FACL4; ab92501, Abcam), mitochondrial fission 1 (FIS1; ab156865, Abcam), calnexin (ab133615, Abcam), microtubule-associated protein 1 light chain 3 beta (MAP1LC3B; ab192890, Abcam), translocase of outer mitochondrial membrane 20 (TOMM20; ab186735, Abcam), and actin beta (ACTB; ab8226, Abcam). Protein bands were visualized by chemiluminescence detection and quantified using the Image QuantTL software (GE Healthcare, Sweden).

Measurement of mitochondrial calcium in intact cells. Cardiomyocytes were seeded on glass-bottomed cell culture dishes and incubated with $1 \mu \mathrm{M}$ of the calcium indicator Rhod2AM (ab142780, Abcam) at $37^{\circ} \mathrm{C}$ in the dark for $30 \mathrm{~min}$, as per the manufacturer's guidelines. Next, the cells were washed twice with calcium-free HBSS and imaged under a laser scanning confocal microscope (LSCM, Leica TCS-SP5). The fluorescence intensity $(F)$ was normalized to the baseline fluorescence value $F_{0}\left(F / F_{0}\right)$ and expressed as mitochondrial calcium concentration $\left(\left[\mathrm{Ca}^{2+}\right]_{\mathrm{m}}\right)$. We measured $\mathrm{F}_{\max }$ and $\mathrm{F}_{\min }$, as previously described ${ }^{50}$. $\mathrm{F}_{\text {max }}$ was obtained by perfusion with $10 \mu \mathrm{M}$ ionomycin and $5 \mathrm{mM} \mathrm{CaCl}_{2} ; \mathrm{F}_{\min }$ was measured by perfusion with $10 \mathrm{mM}$ EGTA and $20 \mu \mathrm{M}$ BAPTA-AM (B1205, Molecular probes) in HBSS. 2-APB (ab120124, Abcam), TG (T9033, Sigma-Aldrich), and ATP (A1852, Sigma-Aldrich) were also added to the external solution at a proper final concentration. The fluorescence intensity of Rhod2-AM was measured using LSCM. The fluorescence intensity was converted to $\left[\mathrm{Ca}^{2+}\right]$ using the following formula: $\left[\mathrm{Ca}^{2+}\right]_{\mathrm{m}}=K_{\mathrm{d}} \times\left(\mathrm{F}-\mathrm{F}_{\mathrm{min}}\right) /$ $\left(\mathrm{F}_{\max }-\mathrm{F}\right)$, where $K_{\mathrm{d}}$ is the equilibrium dissociation constant of Rhod 2 for $\mathrm{Ca}^{2+}$, which was $570 \mathrm{nM}$.

Immunofluorescence. Cardiomyocytes were stained with MitoTracker Deep Red FM (500 nM; M22426, Invitrogen) and fixed in 4\% paraformaldehyde (P0099, Beyotime Institute of Biotechnology) at room temperature for $10 \mathrm{~min}$. They were then permeabilized with 0.1\% Triton 100-X (P0096, Beyotime Institute of Biotechnology) at room temperature for $30 \mathrm{~min}$. Cells were washed with phosphate-buffered saline (PBS) three times and blocked in blocking buffer (P0102, Beyotime Institute of Biotechnology) for immunostaining at $37^{\circ} \mathrm{C}$ for $30 \mathrm{~min}$. Samples were incubated with anti-MAP1LC3B antibody (1:100) or anti-ERP72 antibody (ab155800, Abcam; $1: 100)$ at $4^{\circ} \mathrm{C}$ overnight and then washed in PBS twice, before staining with the secondary antibody $(31561$, Invitrogen; $1: 500)$ at $37^{\circ} \mathrm{C}$ for $2 \mathrm{~h}$. Co-localization of fluorescence was measured at $100-400 \mathrm{~Hz}$ under the LSCM. Samples without primary antibodies were used as negative controls. Images were analyzed using LAS X software (Leica) and Image-Pro Plus 5.0 (Media Cybernetics). Co-localization represented in Pearson's correlation coefficient was measured using automatic thresholding, as previously described ${ }^{51}$. 
Measurement of mitochondrial bioenergetics and FAO metabolism. The cellular oxygen consumption rate (OCR) and extracellular acidification rate (ECAR) were measured using a Seahorse XF96 extracellular flux analyzer (Seahorse Bioscience, North Billerica, USA). Briefly, cells with/without HH exposure or transfected with LVVs-Pacs2 were plated in XF96-well microplates (6000 cells/well, Seahorse Bioscience). After reaching the proper cell density, the cells were incubated with XF assay medium without $\mathrm{CO}_{2}$ at $37^{\circ} \mathrm{C}$ for $1 \mathrm{~h}$. For OCR measurement, cells were then serially exposed to $1 \mu \mathrm{M}$ oligomycin (mitochondrial/ATP synthase inhibitor), $2 \mu \mathrm{M}$ of trifluoromethoxy carbonyl cyanide phenylhydrazone (FCCP, a mitochondrial uncoupler), and $0.5 \mu \mathrm{M}$ of rotenone/antimycin $\mathrm{A}$ (respiratory chain inhibitor), provided in the XF Cell Mito Stress Test Kit (Seahorse Bioscience). Three measurements were performed for each cycle (4 min mixing, followed by 3 min detection). Basal respiration, maximal respiration, proton respiration, and coupled respiration were collected using Seahorse XF Extracellular Flux analyzer software following the manufacturer's protocol. To measure the FAO, cells were cultured replaced by FAO assay medium containing palmitate-BSA according to the manufacturer's instructions. Other conditions were consistent with the normal OCR measurement. OCR or ECAR experiments were conducted at $37{ }^{\circ} \mathrm{C}$ and adjusted $\mathrm{pH}$ to 7.4. Following an XF assay, the number of cells were determined and used to normalize OCR and ECAR.

Measurement of mitophagy levels using the mitochondria-targeted Keima reporter. We used the mtKeima reporter to measure the mitophagy levels. Cardiomyocytes were transfected with mitochondria-targeted monomeric Keima-Red-hyg (mtKeima; AM-V0251HM, Medical and Biological Laboratories Co., Ltd.), which contained hygromycin B-resistance gene. Hygromycin B infection was used to screen and obtain cardiomyocytes stably expressing mtKeima; cardiomyocytes were seeded on glass-bottom dishes and observed under an LSCM to evaluate mitophagy levels. The wavelengths of excitation and emission filters used were as follows: cytoplasmic Keima: 488 nm, 650-760 nm, lysosomal Keima: 561 nm, 570$630 \mathrm{~nm}^{52}$. Images were analyzed using ImageJ software. Briefly, the cardiomyocytes and mtKeima were segmented, and the areas of cytoplasmic and lysosomal mtKeima were determined. The mitophagy index was calculated as the ratio of the total area of lysosomal mitochondria to the total area of cytoplasmic mitochondria per well.

Transmission electron microscopy. The right myocardium or $\mathrm{H} 9 \mathrm{C} 2$ cardiomyocytes were fixed in $2.5 \%$ glutaraldehyde for $2 \mathrm{~h}$ and immersed in $1 \%$ osmic acid for $2 \mathrm{~h}$ at $4^{\circ} \mathrm{C}$. The fixed samples were then washed in PBS, dehydrated in a graded series of ethanol. Subsequently, the samples were embedded in Epon 812 (SPI Supplies, West Chester, PA, USA) and placed in a model for polymerization. After the semi thin section was used for positioning, the ultrathin section was made and collected for microstructure analysis, followed by counterstaining with $3 \%$ uranyl acetate and $2.7 \%$ lead citrate. Next, we observed the sections using a HT7800 TEM (HITACHI, Tokyo, Japan) operating at $100 \mathrm{kV}$.

LC-MS metabolomics analysis. We weighed $60 \mathrm{mg}$ of sample and added $20 \mu \mathrm{L}$ of 2-chloro-l-

phenylalanine $(0.3 \mathrm{mg} / \mathrm{mL}$, dissolved in methanol) and $0.6 \mathrm{~mL}$ of mixed solution (methanol/water $=7 / 3$ $(\mathrm{v}: \mathrm{v}))$ into the $1.5 \mathrm{~mL}$ EP tube. The samples were homogenized for $2 \mathrm{~min}$ and then extracted $30 \mathrm{~min}$ by sonication. They were then placed at $-20^{\circ} \mathrm{C}$ for $20 \mathrm{~min}$ and centrifuged at $13000 \mathrm{~g}$ for $15 \mathrm{~min}\left(4^{\circ} \mathrm{C}\right)$. LC- 
HRMS was performed on a Waters UPLC I-class system equipped with a binary solvent delivery manager and a sample manager, coupled with a Waters VION IMS Q-TOF Mass Spectrometer equipped with an electrospray interface (Waters Corporation, Milford, USA). The injection volume was $3.00 \mu \mathrm{L}$, and the column temperature was set at $45^{\circ} \mathrm{C}$. The mass spectrometric data was collected using a Waters VION IMS Q-TOF Mass Spectrometer equipped with an electrospray ionization source operating in either positive or negative ion mode. The source and desolvation temperatures were set at $120^{\circ} \mathrm{C}$ and $500^{\circ} \mathrm{C}$, respectively, with a desolvation gas flow of $900 \mathrm{~L} / \mathrm{h}$. Centroid data was collected from 50 to $1000 \mathrm{~m} / \mathrm{z}$ with a scan time of $0.1 \mathrm{~s}$ and an interscan delay of $0.02 \mathrm{~s}$ over a 13-min analysis time.

iTRAQ proteomics analysis/nanoUHPLC-MS/MS analysis. Lysis buffer (1\% SDS, 8 M urea, 1x Protease Inhibitor Cocktail [Roche Ltd. Basel, Switzerland]) was added into the samples and vibrated and milled for $400 \mathrm{~s}$ thrice. The samples were then lysed on ice for $30 \mathrm{~min}$ and centrifuged at $15000 \mathrm{rpm}$ for $15 \mathrm{~min}$ at $4^{\circ} \mathrm{C}$. The protein concentration of the supernatant was determined using the BCA protein assay; we then transferred $100 \mu \mathrm{g}$ of protein/condition into a new Eppendorf tube and adjusted the final volume to 100 $\mu \mathrm{L}$ with $8 \mathrm{M}$ urea. We added $2 \mu \mathrm{L}$ of $0.5 \mathrm{M}$ TCEP and incubated the sample at $37^{\circ} \mathrm{C}$ for $1 \mathrm{~h}$; next, $4 \mu \mathrm{L}$ of 1 $\mathrm{M}$ iodoacetamide was added to the sample, and the incubation lasted 40 min protected from light at room temperature. Five volumes of $-20^{\circ} \mathrm{C}$ pre-chilled acetone were then added to precipitate the proteins overnight at $-20^{\circ} \mathrm{C}$. The precipitates were washed by $1-\mathrm{mL}$ pre-chilled $90 \%$ acetone aqueous solution twice and then re-dissolved in $100 \mu \mathrm{L} 100 \mathrm{mM}$ TEAB. Sequence grade modified trypsin (Promega, Madison, $\mathrm{WI}$ ) was added at the ratio of 1:50 (enzyme: protein, weight: weight) to digest the proteins at $37^{\circ} \mathrm{C}$ overnight. The peptide mixture was desalted by C18 ZipTip, quantified by Pierce ${ }^{\text {TM }}$ Quantitative Colorimetric Peptide Assay (23275), and lyophilized by SpeedVac.

The resultant peptide mixture was labeled with iTRAQ 8Plex labeling kit (Sciex) following the manufacturer's instructions. The labeled peptide samples were then pooled and lyophilized in a vacuum concentrator. The peptide mixture was re-dissolved in the buffer A ( $20 \mathrm{mM}$ ammonium formate in water, $\mathrm{pH}$ 10.0, adjusted with ammonium hydroxide), and then fractionated by high $\mathrm{pH}$ separation using Ultimate 3000 system (ThermoFisher Scientific, MA, USA) connected to a reverse-phase column (XBridge C18 column, Waters Corporation, MA, USA). High pH separation was performed using a linear gradient, starting from 5\% B to $45 \% \mathrm{~B}$ in 40 min (B: $20 \mathrm{mM}$ ammonium formate in $80 \% \mathrm{ACN}, \mathrm{pH} 10.0$, adjusted with ammonium hydroxide). The peptides were re-dissolved in $5 \% \mathrm{ACN}$ aqueous solution containing $0.5 \%$ formic acid and analyzed by on-line nanospray LC-MS/MS on Q Exactive ${ }^{\text {TM }} \mathrm{HF}-\mathrm{X}$ coupled to EASY-nLC 1200 system (Thermo Fisher Scientific, MA, USA). The column flow rate was maintained at $250 \mathrm{~nL} / \mathrm{min}$. The electrospray voltage of $2 \mathrm{kV}$ versus the inlet of the mass spectrometer was used.

Bioinformatics data analysis. The UPLC-Q-TOF/MS raw data were analyzed using progenesis QI (Waters CorporationMilford, USA) software. The parameters used were retention time (RT) range 0.5-14.0 min, mass range 50-1000 Da, and mass tolerance 0.01 Da. Isotopic peaks were excluded from the analysis, the noise elimination level was set at 10.00 , the minimum intensity was set to $15 \%$ of base peak intensity, and RT tolerance was set at $0.01 \mathrm{~min}$. The Excel file was obtained with three-dimension data sets including $\mathrm{m} / \mathrm{z}$, peak RT and peak intensities; RT-m/z pairs were used as the identifier for each ion. The 
resulting matrix was further reduced by removing any peaks with missing values (ion intensity $=0$ ) in > $60 \%$ of samples. The internal standard was used for data QC (reproducibility). The positive and negative data were combined to yield a combined data set imported into SIMCA-P + 14.0 software package (Umetrics, Umeå, Sweden). Principle component analysis and (orthogonal) partial least-squaresdiscriminant analysis ([O] PLS-DA) were performed to visualize the metabolic alterations among the experimental groups, after mean centering and unit variance scaling. Tandem mass spectra were processed by PEAKS Studio version X (Bioinformatics Solutions Inc., Waterloo, Canada). Differentially expressed proteins were filtered if they contained $\geq 1$ unique peptide with $P \leq 0.05$ and fold change $\geq$ 1.2. The pathway analysis was performed using Gene Ontology (GO) and the Kyoto Encyclopedia of Genes and Genomes (KEGG) database.

Statistical analysis and reproducibility. All statistical analyses were performed with SPSS 20.0 software. The measurement variables were presented as mean \pm standard deviation (SD) in minimum triplicates. Statistical significance was determined using Student's $t$-test between two groups and corrected for multiple comparisons (Least-Significant Difference) for more than two groups. Mann-Whiney test or nonparametric ANOVA (Kruskal-Wallis) followed by the Dunn multiple comparison post-hoc test was used when one or more datasets showed non-normal distribution. Imaging experiments and animal tests were assessed in a blinded fashion. Sample sizes were kept similar between experimental groups and replicates of experiments. The number of biological replicates and observations are described in the figure legends. Statistical significance was considered at $P<0.05$, with $* P<0.05$, ${ }^{\star *} P<0.01$. For graphs, all data were analyzed using GraphPad Prism software (version 5.0 or 8.4.0).

\section{Data Availability}

All data supports the main and supplementary figures are either available online or available from the corresponding authors upon reasonable request.

\section{Declarations}

\section{Competing interests}

The authors declare no competing interests.

\section{Additional information}

Supplementary information is available for this paper.

\section{References}


1. Maufrais, C. et al. Medex 2015: The key role of cardiac mechanics to maintain biventricular function at high altitude. Exp. Physiol. 104, 667-676 (2019).

2. Osculati, G. et al. Effects of hypobaric hypoxia exposure at high altitude on left ventricular twist in healthy subjects: data from HIGHCARE study on Mount Everest. Eur. Heart J. Cardiovasc. Imaging 17, 635-643 (2016).

3. Stembridge, M., Ainslie, P. N. \& Shave, R. Short-term adaptation and chronic cardiac remodelling to high altitude in lowlander natives and Himalayan Sherpa. Exp. Physiol. 100, 1242-1246 (2015).

4. Rezaie, J. et al. Cardioprotective role of extracellular vesicles: A highlight on exosome beneficial effects in cardiovascular diseases. J. Cell. Physiol. 234, 21732-21745 (2019).

5. Cardoso, A. C. et al. Mitochondrial Substrate Utilization Regulates Cardiomyocyte Cell Cycle Progression. Nat. Metab. 2, 167-178 (2020).

6. Biniecka, M. et al. Hypoxia induces mitochondrial mutagenesis and dysfunction in inflammatory arthritis. Arthritis Rheum. 63, 2172-2182 (2011).

7. Giordano, F. J. Oxygen, oxidative stress, hypoxia, and heart failure. J. Clin. Invest. 115, 500-508 (2005).

8. Liu, L. et al. Mitochondrial outer-membrane protein FUNDC1 mediates hypoxia-induced mitophagy in mammalian cells. Nat. Cell Biol. 14, 177-185 (2012).

9. MacVicar, T. D., Mannack, L. V., Lees, R. M. \& Lane, J. D. Targeted siRNA Screens Identify ER-toMitochondrial Calcium Exchange in Autophagy and Mitophagy Responses in RPE1 Cells. Int. J. Mol. Sci. 16, 13356-13380 (2015).

10. Tong, M. et al. Mitophagy Is Essential for Maintaining Cardiac Function During High Fat Diet-Induced Diabetic Cardiomyopathy. Circ. Res. 124, 1360-1371 (2019).

11. Wu, H. \& Chen, Q. Hypoxia activation of mitophagy and its role in disease pathogenesis. Antioxid. Redox Signal. 22, 1032-1046 (2015).

12. Johansen, T. \& Lamark, T. Selective Autophagy: ATG8 Family Proteins, LIR Motifs and Cargo Receptors. J. Mol. Biol. 432, 80-103 (2020).

13. Csordás, G. et al. Imaging interorganelle contacts and local calcium dynamics at the ERmitochondrial interface. Mol. Cell 39, 121-132 (2010).

14. Rowland, A. A. \& Voeltz, G. K. Endoplasmic reticulum-mitochondria contacts: function of the junction. Nat. Rev. Mol. Cell Biol. 13, 607-625 (2012).

15. Gomez-Suaga, P. et al. The ER-Mitochondria Tethering Complex VAPB-PTPIP51 Regulates Autophagy. Curr. Biol. 27, 371-385 (2017).

16. Böckler, S. \& Westermann, B. ER-mitochondria contacts as sites of mitophagosome formation. Autophagy 10, 1346-1347 (2014).

17. Fan, Y. \& Simmen, T. Mechanistic Connections between Endoplasmic Reticulum (ER) Redox Control and Mitochondrial Metabolism. Cells 8 (2019). 
18. Dombernowsky, S. L. et al. The sorting protein PACS-2 promotes ErbB signalling by regulating recycling of the metalloproteinase ADAM17. Nat. Commun. 6, 7518 (2015).

19. Aslan, J. E. et al. Akt and 14-3-3 control a PACS-2 homeostatic switch that integrates membrane traffic with TRAIL-induced apoptosis. Mol. Cell 34, 497-509 (2009).

20. Kveiborg, M. \& Thomas, G. PACS-2 functions in colorectal cancer. Aging (Albany NY) 10, 1190-1191 (2018).

21. Simmen, T. et al. PACS-2 controls endoplasmic reticulum-mitochondria communication and Bidmediated apoptosis. EMBO J. 24, 717-729 (2005).

22. Rizzuto, R. et al. Close contacts with the endoplasmic reticulum as determinants of mitochondrial Ca2+ responses. Science 280, 1763-1766 (1998).

23. Csordás, G. et al. Structural and functional features and significance of the physical linkage between ER and mitochondria. J. Cell Biol. 174, 915-921 (2006).

24. Smith, K. A. et al. Role of Hypoxia-Inducible Factors in Regulating Right Ventricular Function and Remodeling during Chronic Hypoxia-induced Pulmonary Hypertension. Am. J. Respir. Cell Mol. Biol. 63, 652-664 (2020).

25. Boos, C. J. et al. A Four-Way Comparison of Cardiac Function with Normobaric Normoxia, Normobaric Hypoxia, Hypobaric Hypoxia and Genuine High Altitude. PLoS One 11, e0152868 (2016).

26. Roach, R. C., Loeppky, J. A. \& Icenogle, M. V. Acute mountain sickness: increased severity during simulated altitude compared with normobaric hypoxia. J. Appl. Physiol. (1985) 81, 1908-1910 (1996).

27. Savourey, G., Launay, J. C., Besnard, Y., Guinet, A. \& Travers, S. Normo- and hypobaric hypoxia: are there any physiological differences? Eur. J. Appl. Physiol. 89, 122-126 (2003).

28. Wu, L. et al. Effect of HIF-1a/miR-10b-5p/PTEN on Hypoxia-Induced Cardiomyocyte Apoptosis. J. Am. Heart Assoc. 8, e011948 (2019).

29. Kumar, S. et al. HIMF (Hypoxia-Induced Mitogenic Factor)-IL (Interleukin)-6 Signaling Mediates Cardiomyocyte-Fibroblast Crosstalk to Promote Cardiac Hypertrophy and Fibrosis. Hypertension (Dallas, Tex. 1979) 73, 1058-1070 (2019).

30. Werneburg, N. W. et al. Tumor necrosis factor-related apoptosis-inducing ligand (TRAIL) proteininduced lysosomal translocation of proapoptotic effectors is mediated by phosphofurin acidic cluster sorting protein-2 (PACS-2). J. Biol. Chem. 287, 24427-24437 (2012).

31. Wu, S. et al. Binding of FUN14 Domain Containing 1 With Inositol 1,4,5-Trisphosphate Receptor in Mitochondria-Associated Endoplasmic Reticulum Membranes Maintains Mitochondrial Dynamics and Function in Hearts in Vivo. Circulation 136, 2248-2266 (2017).

32. Wu, W. et al. FUNDC1 is a novel mitochondrial-associated-membrane (MAM) protein required for hypoxia-induced mitochondrial fission and mitophagy. Autophagy 12, 1675-1676 (2016).

33. Vásquez-Trincado, C. et al. Mitochondrial dynamics, mitophagy and cardiovascular disease. J. Physiol. 594, 509-525 (2016). 
34. Liu, L., Sakakibara, K., Chen, Q. \& Okamoto, K. Receptor-mediated mitophagy in yeast and mammalian systems. Cell Res. 24, 787-795 (2014).

35. Jia, J. et al. AMPK is activated during lysosomal damage via a galectin-ubiquitin signal transduction system. Autophagy 16, 1550-1552 (2020).

36. Ghislat, G., Patron, M., Rizzuto, R. \& Knecht, E. Withdrawal of essential amino acids increases autophagy by a pathway involving $\mathrm{Ca} 2+$ /calmodulin-dependent kinase kinase- $\beta$ (CaMKK- $\beta$ ). J. Biol. Chem. 287, 38625-38636 (2012).

37. East, D. A. \& Campanella, M. Ca2+ in quality control: an unresolved riddle critical to autophagy and mitophagy. Autophagy 9, 1710-1719 (2013).

38. Hayashi, T. \& Su, T. P. Sigma-1 receptor chaperones at the ER-mitochondrion interface regulate $\mathrm{Ca}(2+)$ signaling and cell survival. Cell 131, 596-610 (2007).

39. Tang, Y. \& Wu, Y. Decreased ATP production during mitochondrial calcium uniporter inhibition enhances autophagy and mitophagy to provide cardioprotection in cardiac failure. Int. J. Cardiol. 282, 67 (2019).

40. McCormack, J. G. \& Denton, R. M. The role of intramitochondrial Ca2+ in the regulation of oxidative phosphorylation in mammalian tissues. Biochem. Soc. Trans. 21, 793-799 (1993).

41. Boyman, L., Karbowski, M. \& Lederer, W. J. Regulation of Mitochondrial ATP Production: $\mathrm{Ca}^{2+}$ Signaling and Quality Control. Trends Mol. Med. 26, 21-39 (2020).

42. Tomar, N. et al. Substrate-dependent differential regulation of mitochondrial bioenergetics in the heart and kidney cortex and outer medulla. Biochim. Biophys. Acta Bioenerg. 1863, 148518 (2021).

43. Denton, R. M., McCormack, J. G. \& Edgell, N. J. Role of calcium ions in the regulation of intramitochondrial metabolism. Effects of $\mathrm{Na}+\mathrm{Mg} 2+$ and ruthenium red on the $\mathrm{Ca} 2+-$ stimulated oxidation of oxoglutarate and on pyruvate dehydrogenase activity in intact rat heart mitochondria. Biochem. J. 190, 107-117 (1980).

44. Brandes, R. \& Bers, D. M. Increased work in cardiac trabeculae causes decreased mitochondrial NADH fluorescence followed by slow recovery. Biophys. J. 71, 1024-1035 (1996).

45. Territo, P. R., Mootha, V. K., French, S. A. \& Balaban, R. S. Ca(2+) activation of heart mitochondrial oxidative phosphorylation: role of the F(0)/F(1)-ATPase. Am. J. Physiol. Cell Physiol. 278, C423-C435 (2000).

46. Murphy, A. N., Kelleher, J. K. \& Fiskum, G. Submicromolar Ca2+ regulates phosphorylating respiration by normal rat liver and AS-30D hepatoma mitochondria by different mechanisms. J. Biol. Chem. 265, 10527-10534 (1990).

47. Baughman, J. M. et al. Integrative genomics identifies MCU as an essential component of the mitochondrial calcium uniporter. Nature 476, 341-345 (2011).

48. Yang, J. et al. Trimetazidine attenuates high-altitude fatigue and cardiorespiratory fitness impairment: A randomized double-blinded placebo-controlled clinical trial. Biomed. Pharmacother. 116, 109003 (2019). 
49. Annunziata, I., Patterson, A. \& d'Azzo, A. Isolation of mitochondria-associated ER membranes (MAMs) and glycosphingolipid-enriched microdomains (GEMs) from brain tissues and neuronal cells. Methods Mol. Biol. 1264, 25-33 (2015).

50. Yang, J. et al. Store-operated calcium entry-activated autophagy protects EPC proliferation via the CAMKK $_{2}$-MTOR pathway in ox-LDL exposure. Autophagy 13, 82-98 (2017).

51. Barlow, A. L., Macleod, A., Noppen, S., Sanderson, J. \& Guérin, C. J. Colocalization analysis in fluorescence micrographs: verification of a more accurate calculation of pearson's correlation coefficient. Microsc. Microanal. 16, 710-724 (2010).

52. Soutar, M. P. M. et al. FBS/BSA media concentration determines CCCP's ability to depolarize mitochondria and activate PINK1-PRKN mitophagy. Autophagy 15, 2002-2011 (2019).

\section{Figures}


a

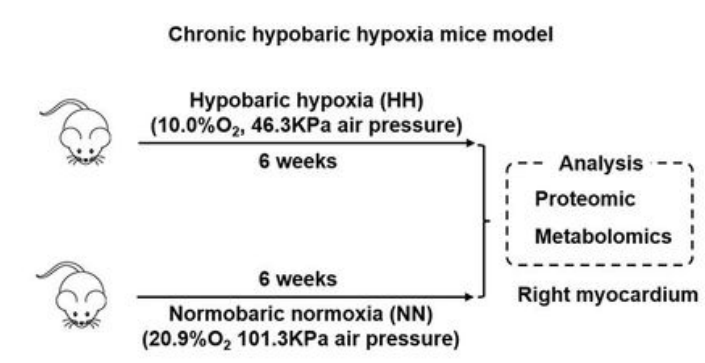

d

\begin{tabular}{cc}
\hline Down-regulated proteins & Fold change \\
\hline PACS2 & -3.16 \\
VPS15 & -2.72 \\
ATG16L & -1.34 \\
MAP1LC3A & -1.29 \\
MAP1LC3B & -1.34 \\
SQSTM1 & -1.26 \\
GABARAP & -1.30 \\
VPS51 & -1.43 \\
RAB8a & -1.26 \\
\hline
\end{tabular}

f

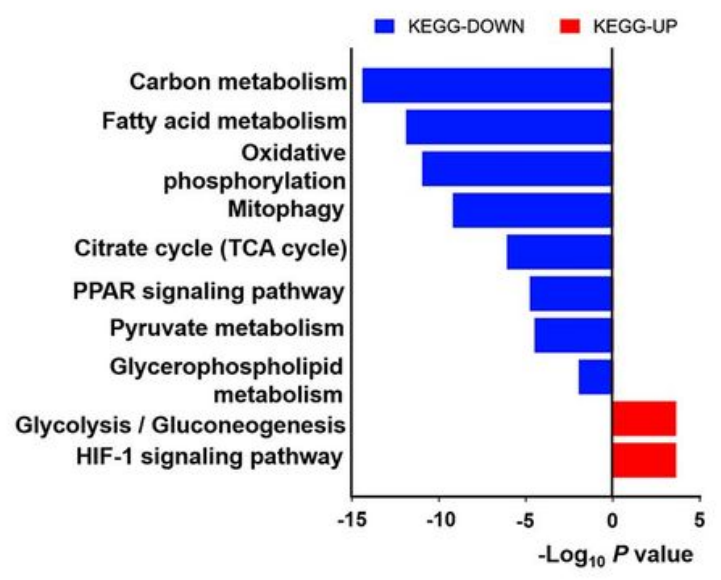

e b

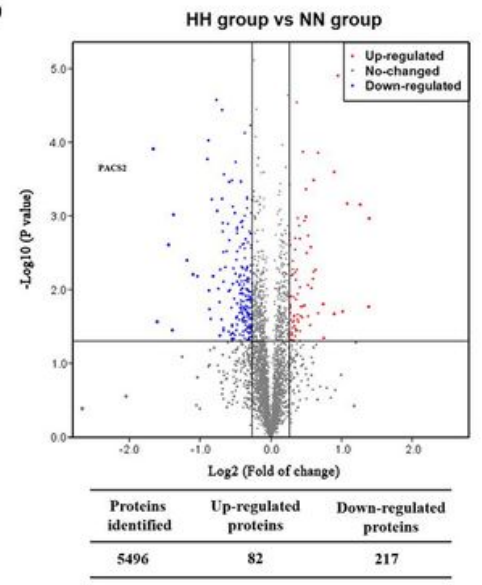

C

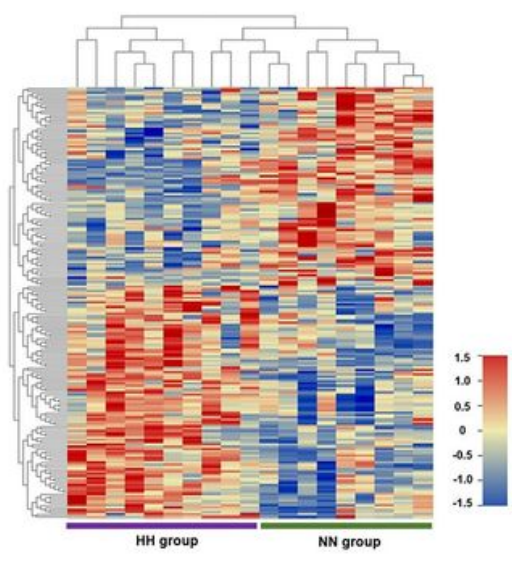

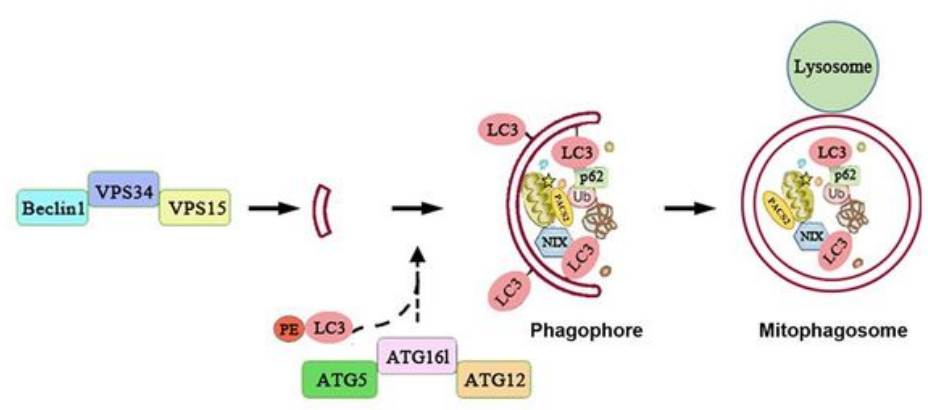

g

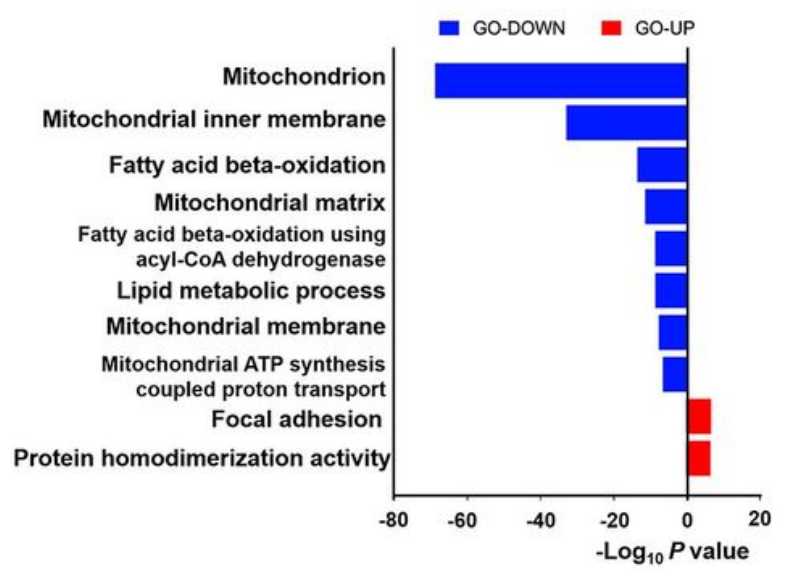

Figure 1

Proteomics and metabolomics assessment of the right myocardium in response to hypobaric hypoxia. a) Protocol for establishing chronic HH-induced mice model and related omics analysis. b) Volcano plot indicating the number of significantly up-regulated (red, $n=82$ ) and down-regulated (blue, $n=217$ ) proteins via proteomics analysis. c) Sample cluster heat map performed with hierarchical clustering in $\mathrm{HH}$ mice heart vs. NN controls are assessed by metabolomics analysis (adjusted $P$ value $<0.05$ ). Red: upregulation and blue: down-regulation. The purple and green labels at the bottom of the heat map represent the separation between the $\mathrm{HH}$ and NN groups. d) Down-regulation of PACS2 (fold change = -3.16) and mitophagy-related proteins in HH group compared with NN controls. e Mechanism diagram of phagophore and mitophagosome formation with the participation of PACS2 and mitophagy-related 
proteins. $\mathbf{f}, \mathbf{g}$ ) Bar graphs of the significantly up-regulated (red) and down-regulated (blue) terms by KEGG (f) and GO (g) pathways analysis. HH, hypobaric hypoxia; NN, normobaric normoxia; KEGG, Kyoto Encyclopedia of Genes and Genomes; GO, gene ontology.

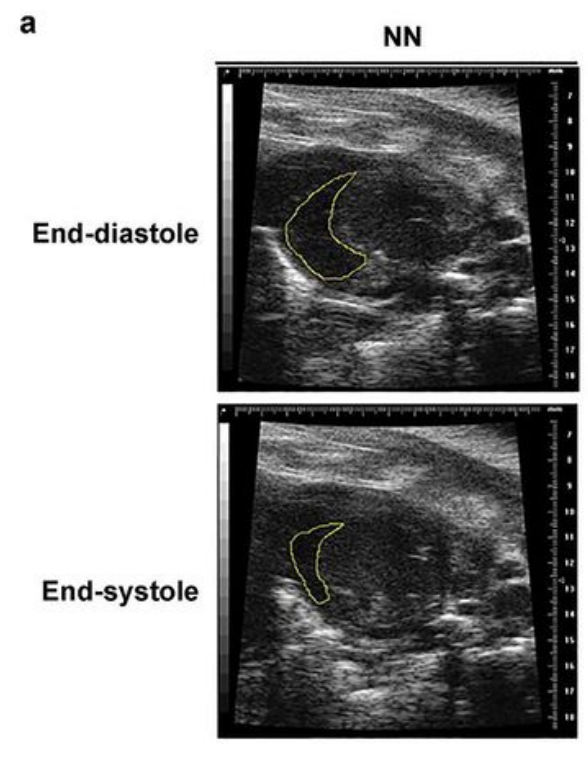

c

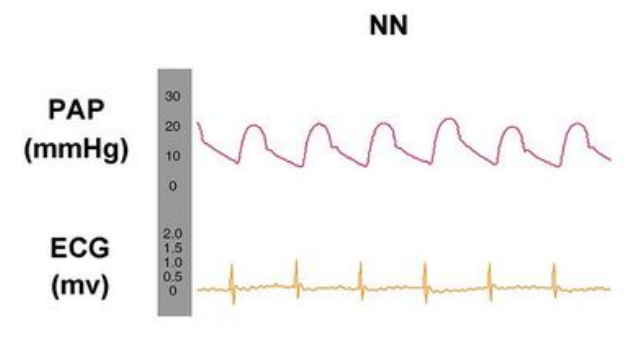

d

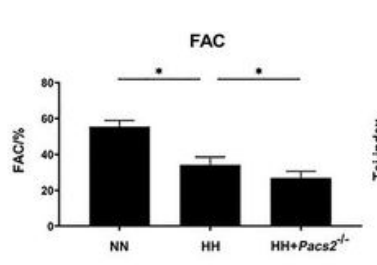

i
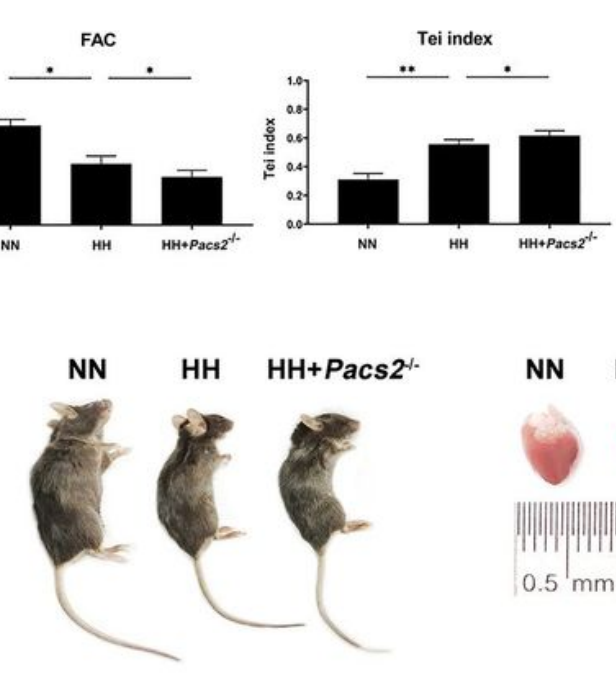

$\mathrm{HH}$

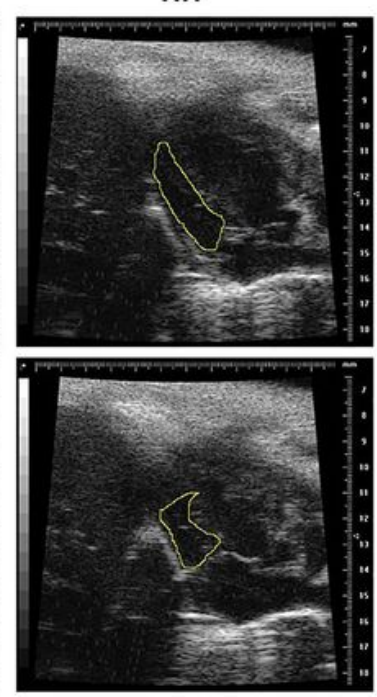

$\mathrm{HH}+$ Pacs $^{--}$

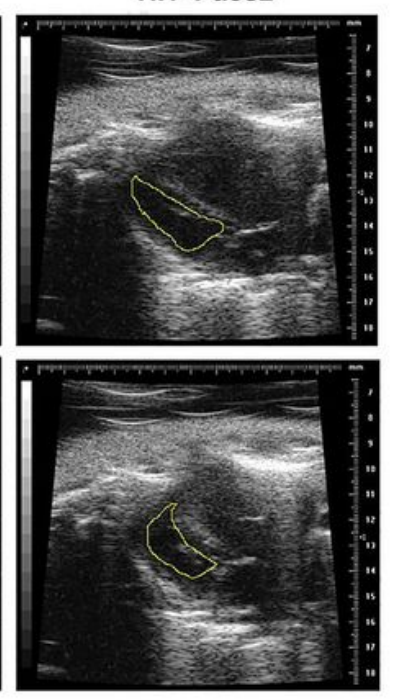

HH b

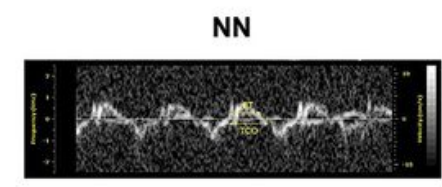

$\mathrm{HH}$

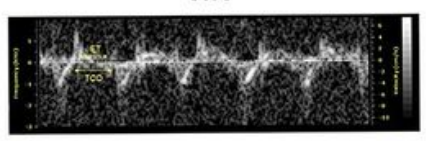

$\mathrm{HH}+$ Pacs2 $^{1}$

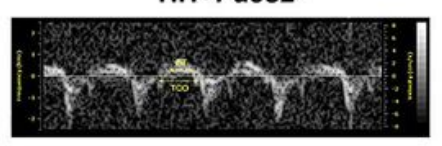

$\mathrm{HH}+$ Pacs $2^{--}$
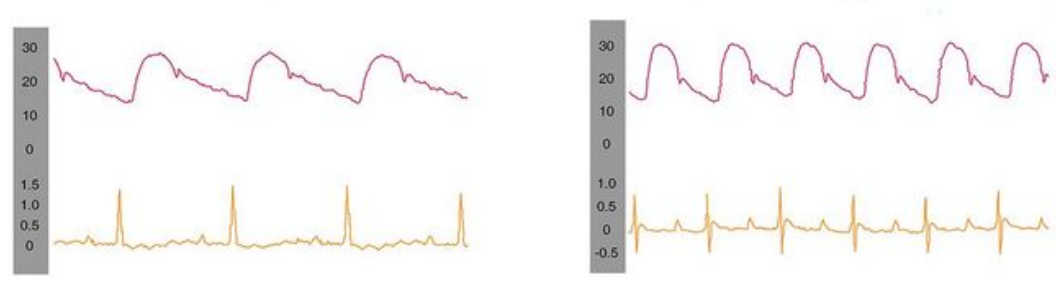

h
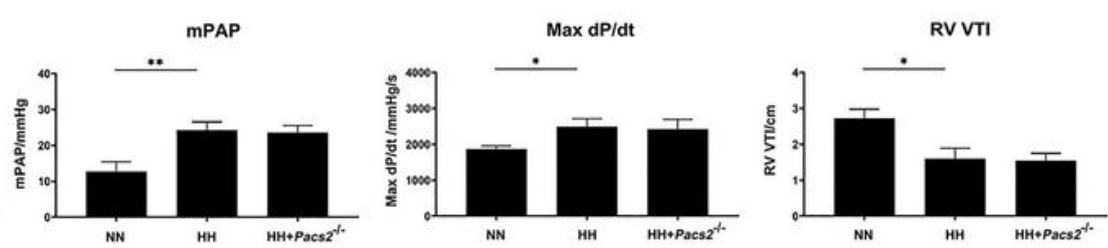

j

k
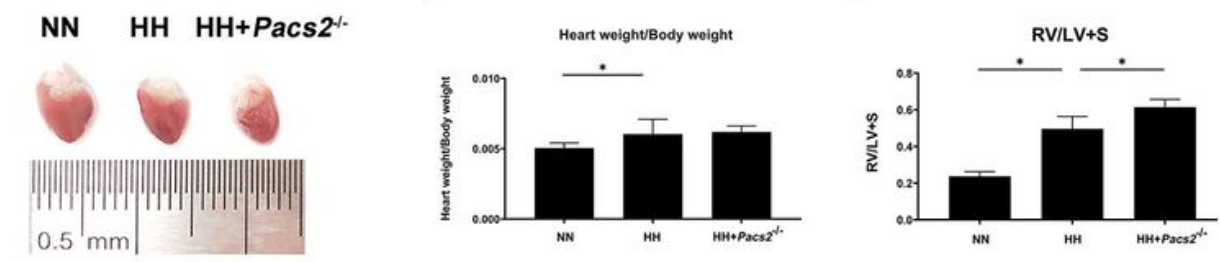

Figure 2

Cardiac Pacs2 ablation exacerbated the right cardiac dysfunction after hypobaric hypoxia. a) FAC measurement of the RV in the NN group (FAC=57.60\%), $\mathrm{HH}$ group $(\mathrm{FAC}=23.36 \%)$, and $\mathrm{HH}+\mathrm{Pacs} 2^{-/-}$group 
( $F A C=18.47 \%$ ). Representative images were acquired at end-diastole (up) and end-systole (down). b) Tei index is measured in the NN (Tei index $=0.35), \mathrm{HH}$ (Tei index=0.50), and $\mathrm{HH}+\mathrm{Pacs}^{-1-}$ groups (Tei index $=0.68$ ) by tissue Doppler imaging. c) mPAP was measured by RHC in the NN (mPAP $=13.27 \mathrm{mmHg}$ ), $\mathrm{HH}(\mathrm{mPAP}=24.67 \mathrm{mmHg})$, and $\mathrm{HH}+\mathrm{Pacs}^{-/-}$groups $(\mathrm{mPAP}=27.66 \mathrm{mmHg}) . \mathbf{d}-\mathbf{h}$ ) Statistics of FAC, Tei index, mPAP, max dP/dt and RV VTI in the three groups are calculated respectively. i) Gross appearance of the whole body (left) and whole heart (right) of the $\mathrm{NN}, \mathrm{HH}$, and $\mathrm{HH}+\mathrm{Pacs}^{-{ }^{-}-}$groups. j, $\mathbf{k}$ ) Bar graphs showing the heart weight to body weight ratio (j) and Fulton's index (k) of the three groups. Cardiac function indexes are obtained from six mice per group. Data was showed by mean $\pm \mathrm{SD}, * P<0.05$, $* * P<$ 0.01. RHC, right heart catheterization; $\mathrm{HH}$, hypobaric hypoxia; NN, normobaric normoxia; RV, right ventricular; $\mathrm{FAC}$, fractional area change; $\max \mathrm{dP} / \mathrm{dt}$, maximum positive time derivative of left ventricular pressure; VTI, velocity time integral; mPAP, mean pulmonary artery pressure. 

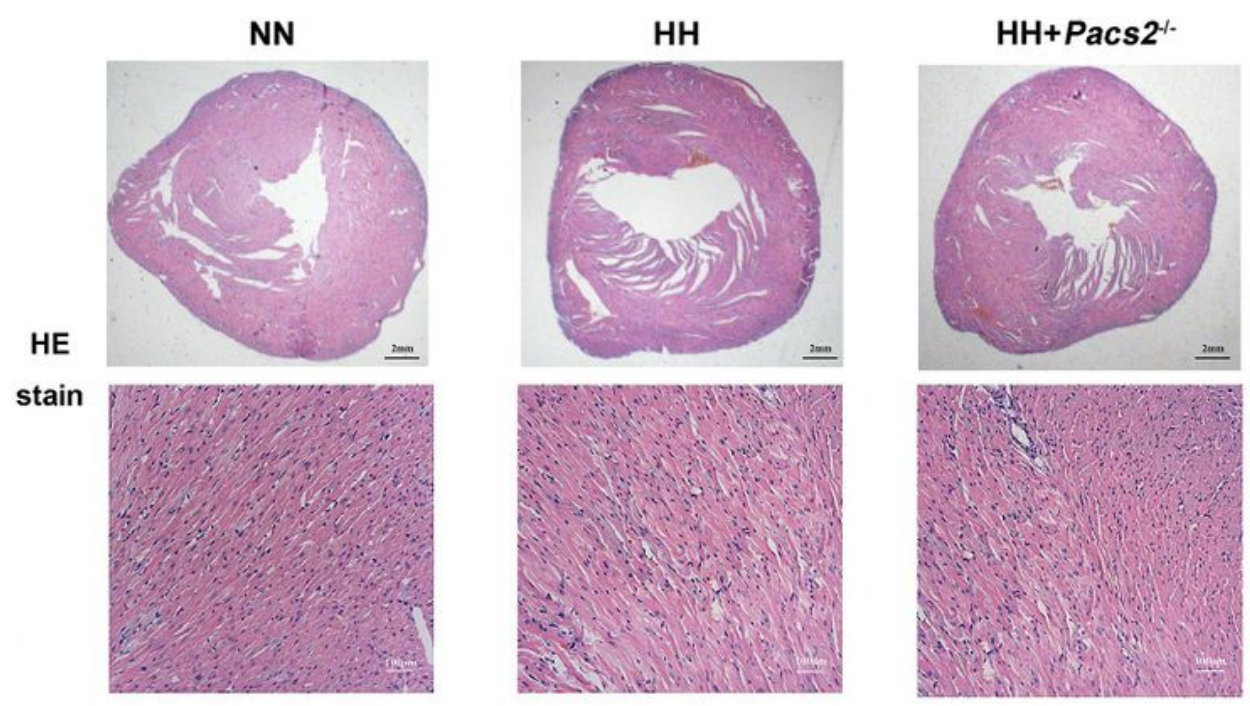

b
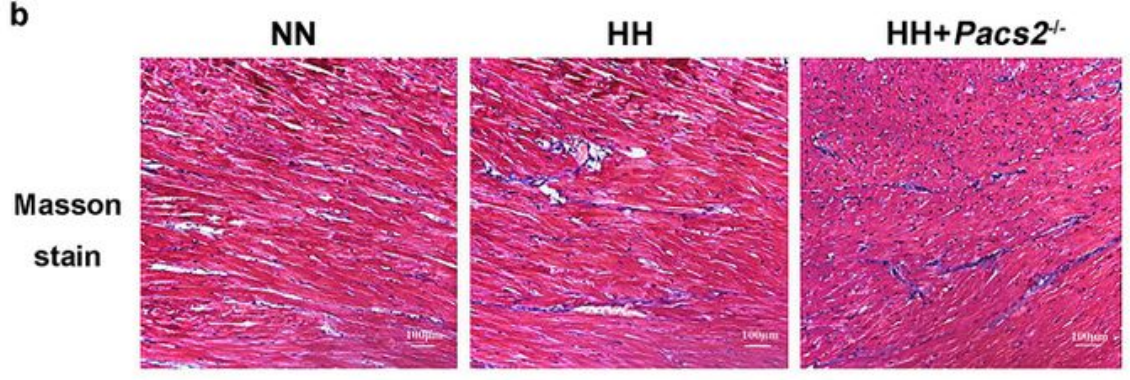

c

NN

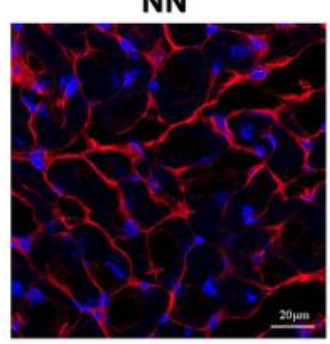

$\mathrm{HH}$

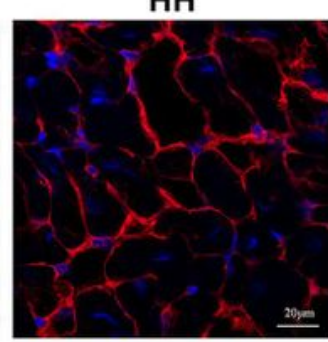

$\mathrm{HH}+$ Pacs $^{-1-}$

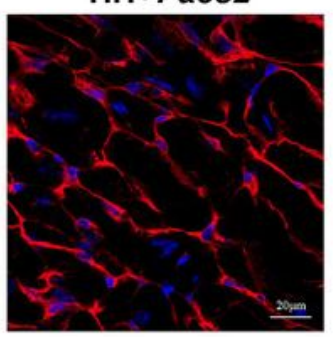

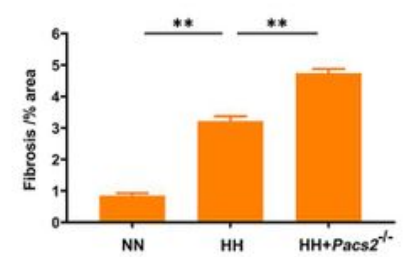

e

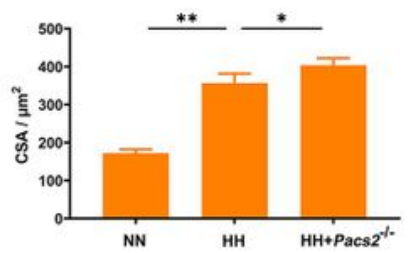

h

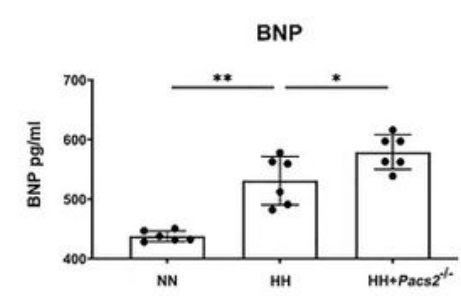

g
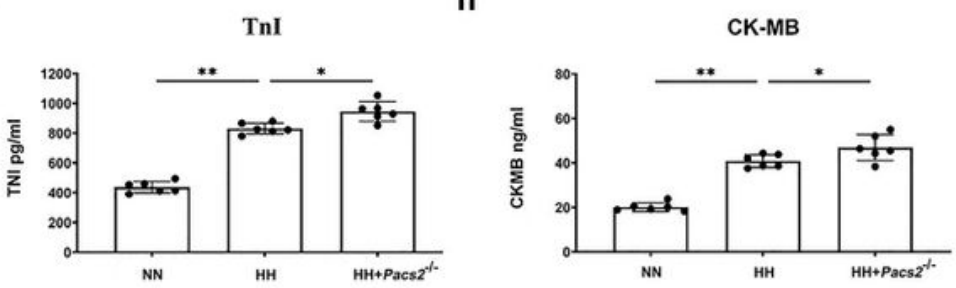

\section{Figure 3}

Cardiac Pacs2 ablation exacerbated the right myocardium structure impairment after hypobaric hypoxia. a-b) Representative HE staining (a) and Masson's trichrome staining (b) images of RV myocardium in NN group, $\mathrm{HH}$ group and $\mathrm{HH}+\mathrm{Pacs}^{-{ }^{--}}$group. Scale bar, $100 \mu \mathrm{m}$. c) Quantification revealing myocardial fibrosis area (blue) in the three groups. d) Representative images of RV tissue stained with WGA (red) to delineate sarcolemma and DAPI (blue). Scale bar, $20 \mu \mathrm{m}$. e) Quantification of relative cardiomyocyte CSA 
in the three groups. $\mathbf{f}-\mathbf{h}$ ) Statistics of plasma concentrations of BNP, TNI and CK-MB of the mice in the three groups. Cardiac function indexes, cardiac tissues and plasma samples are obtained from six mice per group. Data was showed by mean $\pm \mathrm{SD},{ }^{\star} P<0.05,{ }^{\star *} P<0.01$. $\mathrm{HH}$, hypobaric hypoxia; NN, normobaric normoxia; RV, right ventricular; $\mathrm{HE}$, hematoxylin-eosin; WGA, wheat germ agglutinin; CSA, cross-sectional area; BNP, brain natriuretic peptide; TnI, troponin I; CK-MB, creatine kinase MB.
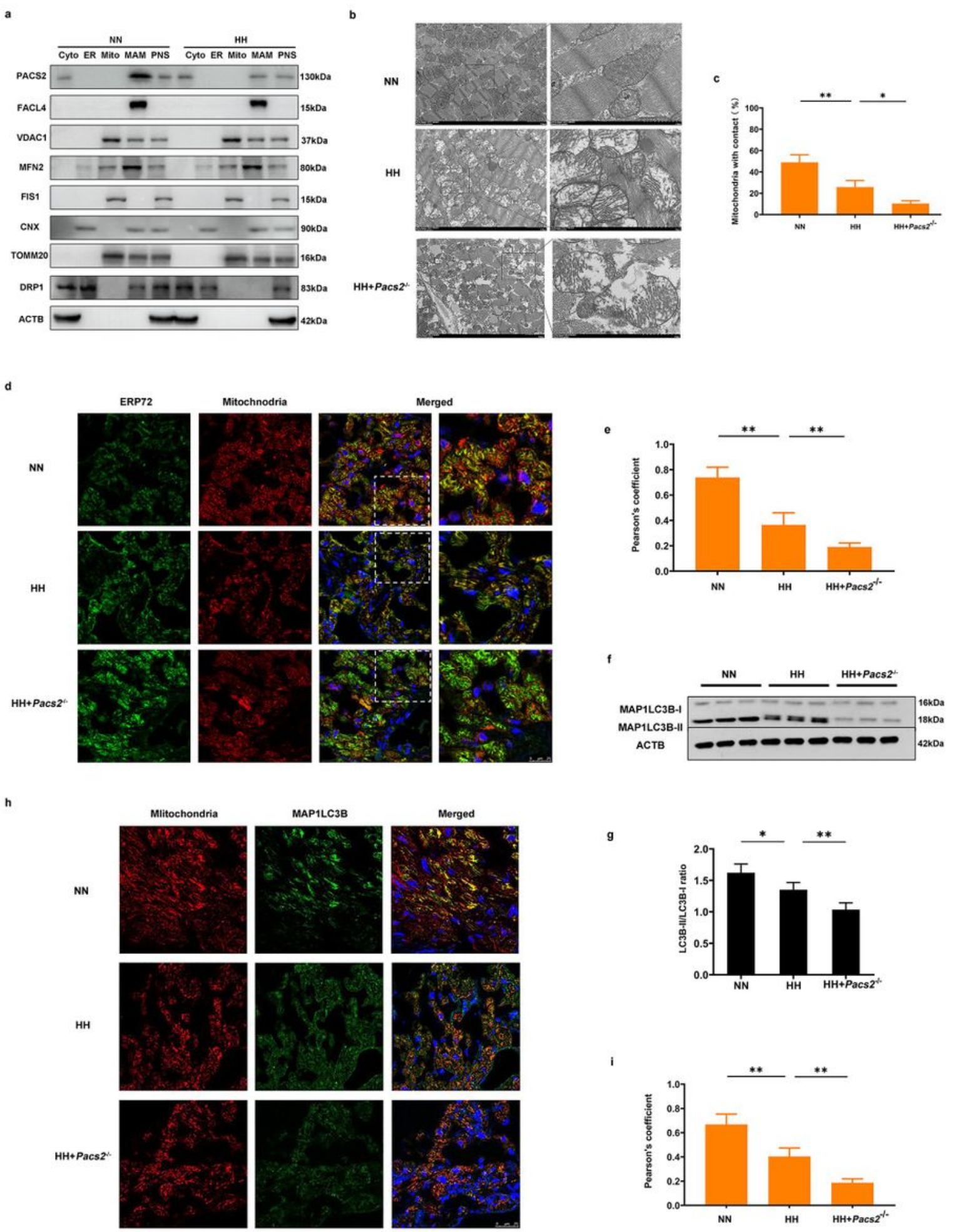

Figure 4 
Cardiac Pacs2 ablation exacerbated MAM disruption and mitophagy reduction induced by hypobaric hypoxia. a) Western blot analysis of PACS2 and MAM-related proteins (FACL4, VDAC1, MFN2, FIS1, CNX, TOMM20 and DRP1) levels in NN and HH conditions. B) Representative TEM images of MAM structure in $\mathrm{NN}, \mathrm{HH}$ and $\mathrm{HH}+\mathrm{Pacs}^{-1}$ group. Scale bar, $2 \mu \mathrm{m}$. c) Quantification of ER and mitochondria contact in the three groups. d) Mitotracker Deep Red is used to mark mitochondria and ERP72 (green) is employed to mark ER in the right myocardium. Scale bar, $25 \mu \mathrm{m}$. e) Pearson's overlap coefficient analysis indicating the lesser co-localization of ER with mitochondria. $\mathbf{f}, \mathbf{g}$ ) Representative western blots and statistical analysis of MAP1LC3B-I, MAP1LC3B-II in the three groups. h) LSCM images of the right myocardium sections which were marked with Mitotracker Deep Red and MAP1LC3B (green). Scale bar, $25 \mu \mathrm{m}$. i) Pearson's overlap coefficient analysis of the co-localization of MAP1LC3B and mitochondria. Right myocardium was obtained from six mice per group. Data are shown as mean $\pm \mathrm{SD},{ }^{*} P<0.05,{ }^{*} P<0.01$. MAM, mitochondria-associated membranes; $\mathrm{HH}$, hypobaric hypoxia; NN, normobaric normoxia; TEM, transmission electron microscopy; FACL, fatty acid CoA ligase 4; VDAC1, voltage-dependent anion channel 1; MFN2, Mitofusin 2, FIS1, Mitochondrial fission 1; CNX, calnexin; TOMM20, translocase of the outer mitochondrial membrane member 20; DRP1, dynamin-related protein 1; ER, endoplasmic reticulum; CYTO, cytosol; MITO, mitochondria; PNS, post-nuclear supernatant; LSCM, laser scanning confocal microscopy.

a
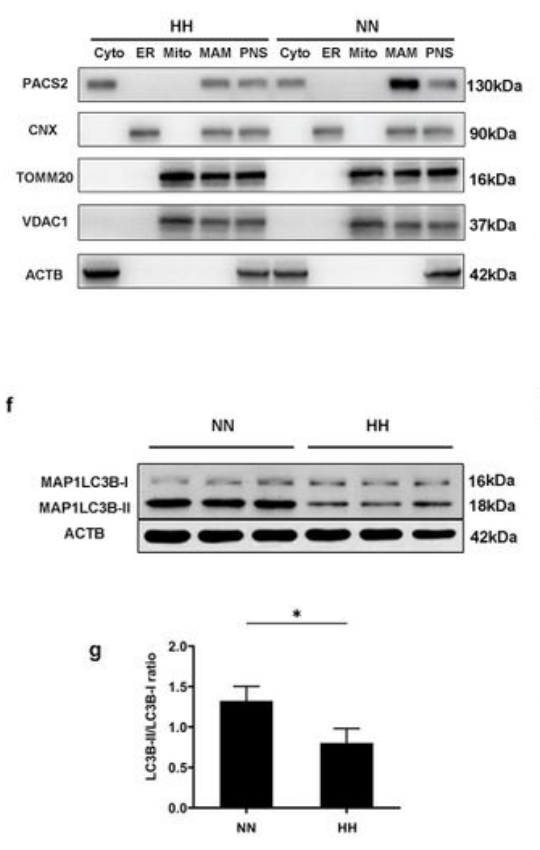
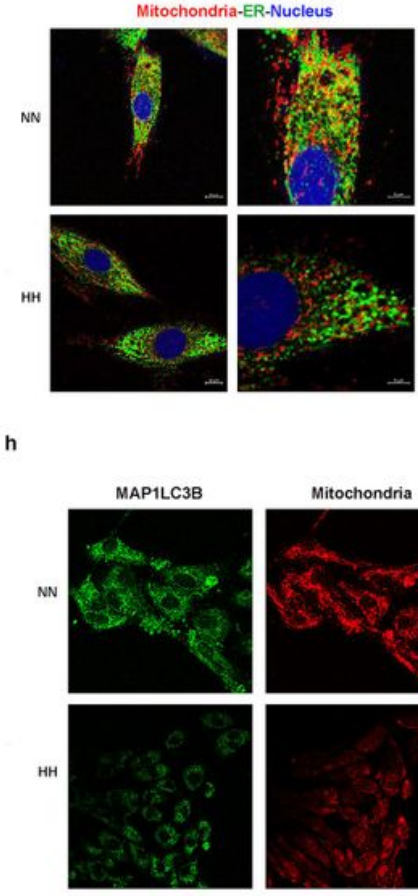
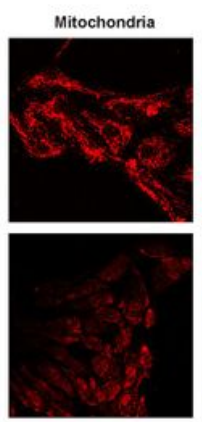

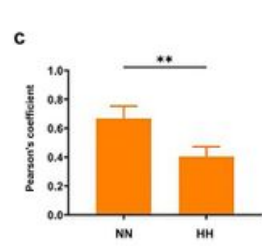

нH

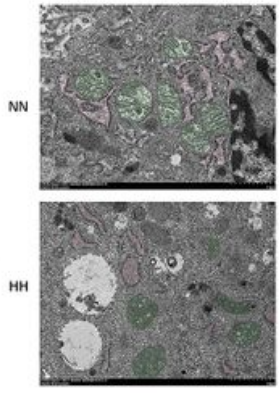

i

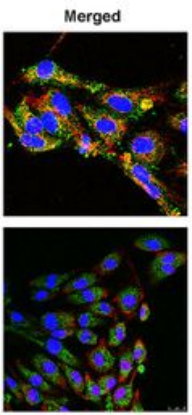

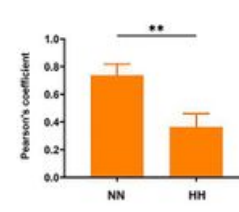

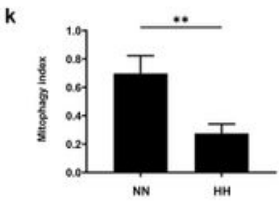

e

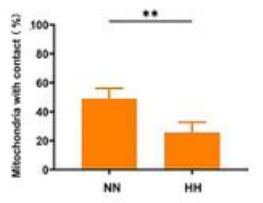

j

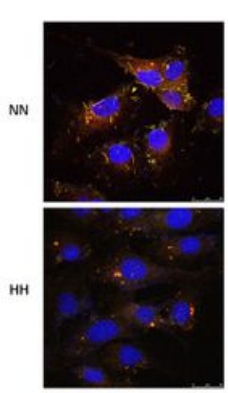

Figure 5

Hypobaric hypoxia reduced PACS2 expression, MAM formation, mitophagy, ER-mitochondria calcium flux and mitochondrial oxidative phosphorylation in vitro. a) Western blot analysis of PACS2 and MAM-related 
proteins (CNX, TOMM2O, VDAC1) in H9C2 cardiomyocytes. b) Cardiomyocytes marked by Mitotracker Deep Red and ERP72 (green) and then analyzed at higher magnification. Scale bar, $5 \mu \mathrm{m}$. c) Pearson's overlap coefficient analysis indicating the lesser co-localization of ER with mitochondria in HH group. $\mathbf{d}$, e) TEM images show lesser mitochondria adjacent to ER in HH treated cardiomyocytes. Scale bar, $1 \mu \mathrm{m}$. f, g) Representative western blots and statistical analysis for MAP1LC3B-I, MAP1LC3B-II under HH and NN exposure. $\mathbf{h}, \mathbf{i})$ LSCM images of cardiomyocytes marked with Mitotracker Deep Red and MAP1LC3B (green). Merged images (h) and Pearson's overlap coefficient analysis (i) revealed that $\mathrm{HH}$ exposure reduced the co-localization of MAP1LC3B and mitochondria. Scale bar, $25 \mu \mathrm{m}$. j, k) H9C2 cardiomyocytes were transfected by mtKeima-red for $12 \mathrm{~h}$ before HH exposure. LSCM images ( $\mathrm{j}$ ) and statistical analysis (k) showed decreased mitophagy index after $\mathrm{HH}$ exposure. Scale bar, $25 \mu \mathrm{m}$. $\mathrm{H} 9 \mathrm{C} 2$ cardiomyocytes were obtained from eight mice per group. Data are shown as mean $\pm \mathrm{SD}$, ${ }^{*} P<0.05,{ }^{*} P<0.01$. $\mathrm{HH}$, simulated hypobaric hypoxia; NN, simulated normobaric normoxia; CNX, calnexin; TOMM20, translocase of the outer mitochondrial membrane member 20; VDAC1, voltage-dependent anion channel 1; ER, endoplasmic reticulum; MAM, mitochondria-associated membranes; TEM, transmission electron microscopy; LSCM, laser scanning confocal microscopy. 
a

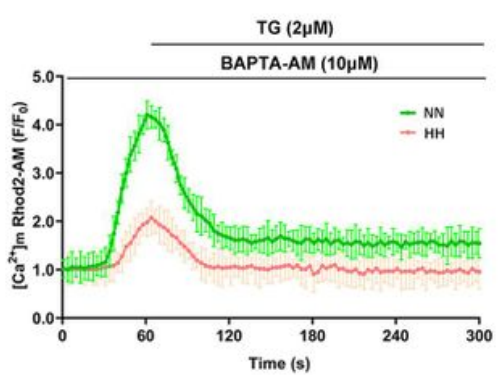

b

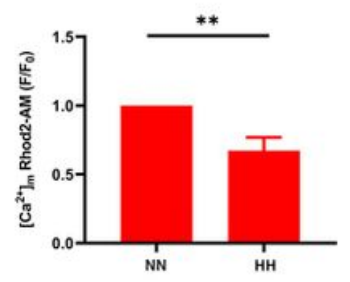

g
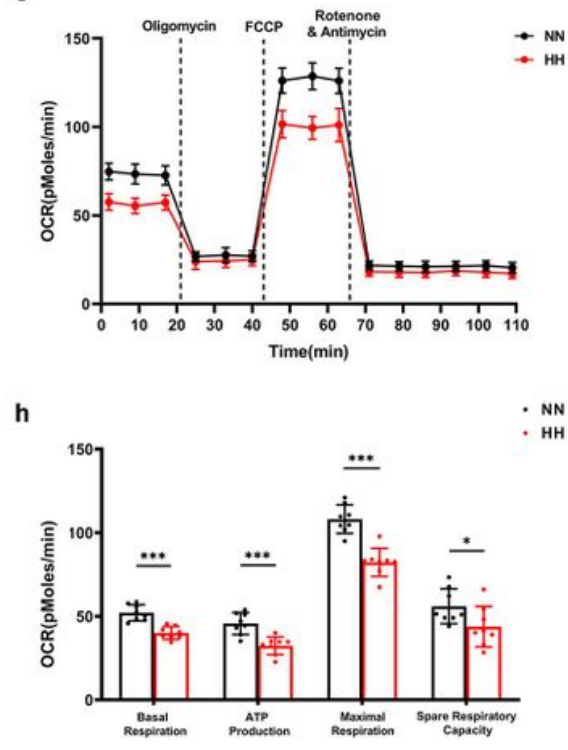

c

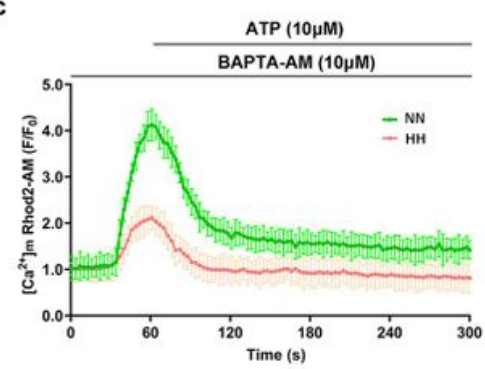

d
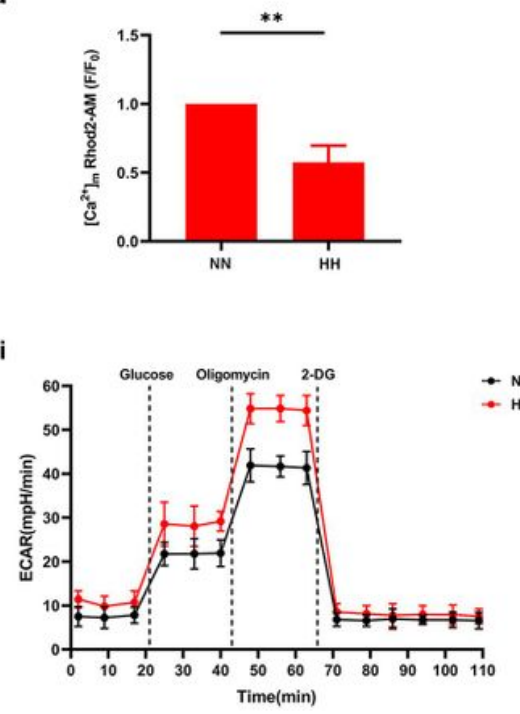

j $\begin{aligned} & \text { NN } \\ & \text {. HH }\end{aligned}$

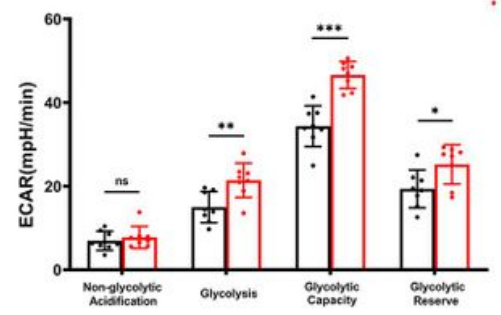

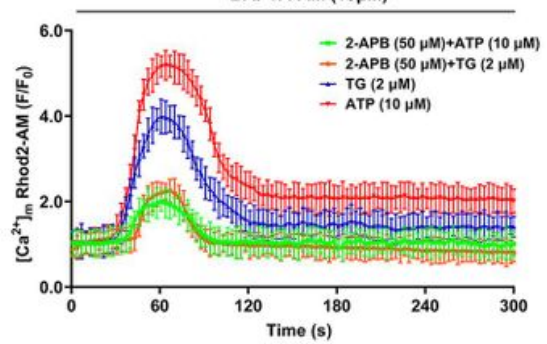
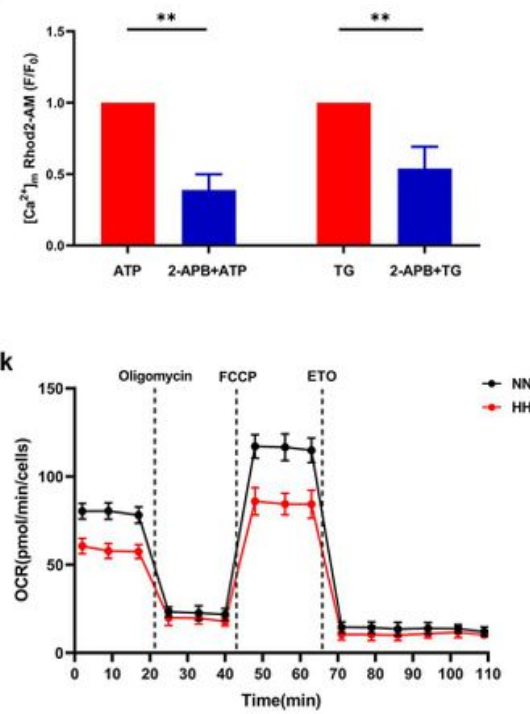

I

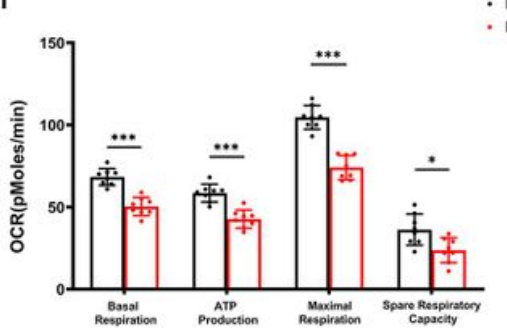

Figure 6

Hypobaric hypoxia reduced ER-mitochondria calcium flux and regulated mitochondrial energy metabolism in vitro. a-d) Cardiomyocytes from NN (green line) and $\mathrm{HH}$ groups (red line) are treated with BAPTA-AM $(10 \mu \mathrm{M})$ for $30 \mathrm{~min}$ prior to and during exposure. Calcium in the mitochondria is stained by Rhod-2 AM probe. Images showing TG $(2 \mu \mathrm{M})(\mathbf{a})$ and ATP $(10 \mu \mathrm{M})(\mathbf{c})$ elicited calcium fluorescence signals. (b, d) Statistical analysis showing relatively lower ER-mitochondria calcium transfer in the $\mathrm{HH}$ group (Cells in NN group were used as controls). e) Quantification of changes in mitochondria calcium concentration in cardiomyocytes pre-treated with 2-APB $(50 \mu \mathrm{M})$ in NN condition. f) Statistical analysis showing decreased mitochondrial calcium flux (Cells without 2-APB treatment are used as controls). $\mathbf{g}-\mathbf{I}$ ) The OCR and ECAR are determined with the Seahorse XF96 Extracellular Flux Analyzer. g) OCR is recorded at baseline and after the sequential injection of each compound (oligomycin, FCCP, rotenone) at the indicated concentration. h) Basal respiration, ATP production, maximal respiration and spare respiratory capacity are calculated. i) ECAR is recorded after the sequential injection of each compound 
(glucose, oligomycin, 2-DG) at the indicated concentration. j) Non-glycolytic acidification, glycolysis, glycolytic capacity and glycolytic reserve are calculated. $\mathbf{k}$ ) OCR analysis of cells treated with BSAconjugated palmitate substrate and after the sequential injection of each compound (oligomycin, FCCP, ETO) at the indicated concentration. I) Basal respiration, ATP production, maximal respiration and spare respiratory capacity are calculated with FAO substrate. $\mathrm{H} 9 \mathrm{C} 2$ cardiomyocytes are obtained from eight mice per group. Cells subjected with or without $\mathrm{HH}$ exposure before loading. Data are shown as mean \pm $\mathrm{SD},{ }^{*} P<0.05,{ }^{\star} P<0.01$. $\mathrm{HH}$, simulated hypobaric hypoxia; NN, simulated normobaric normoxia; laser scanning confocal microscopy; TG, thapsigargin; ATP, adenosine triphosphate; 2-APB, 2aminoethoxydiphenyl borate; OCR, oxygen consumption rate; ECAR, extracellular acidification rate; BAPTA-AM, bis-(aminophenolxy) ethane-N,N,N',N'-tetra-acetic acid acetoxyme-thylester; FCCP, trifluoromethoxy carbonyl cyanide phenylhydrazone; ETO, etomoxir. 


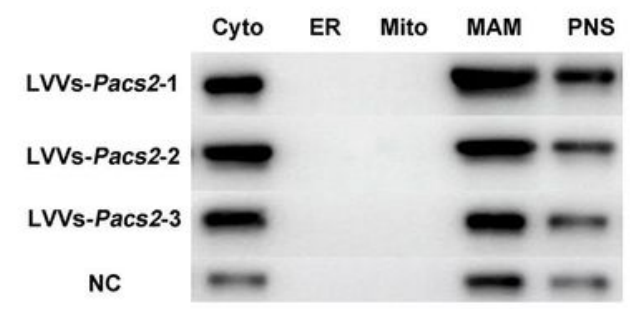

c
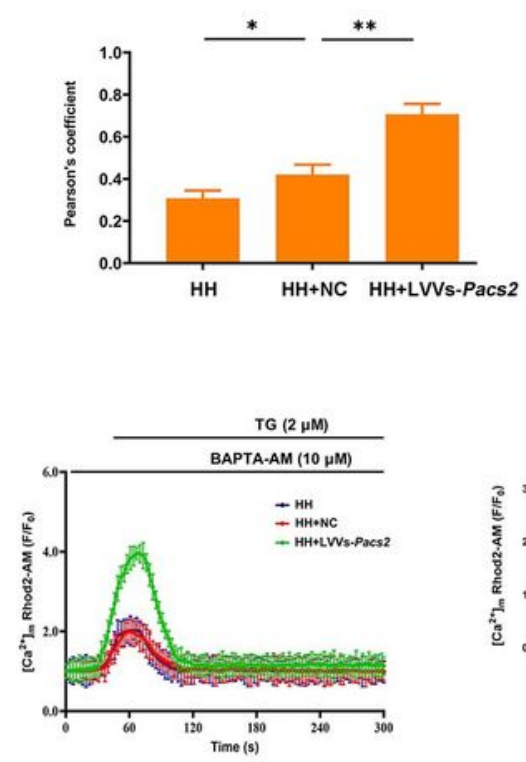

f
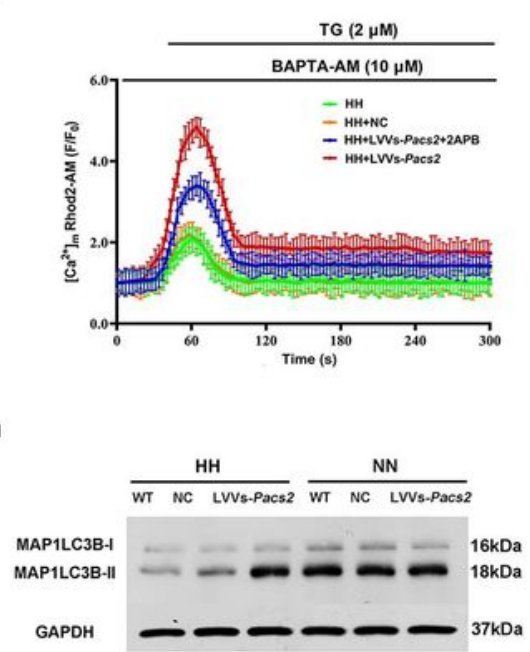

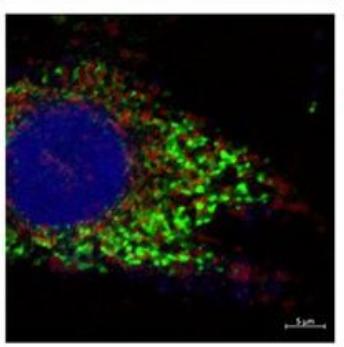

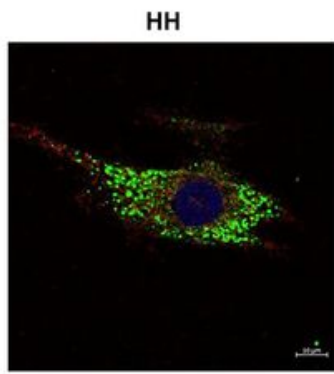

$\mathrm{HH}+\mathrm{NC}$
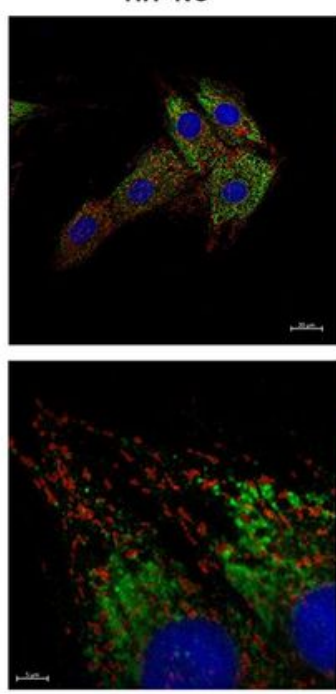

Mitochondria-ER-Nucleus
HH+LVVs-Pacs2
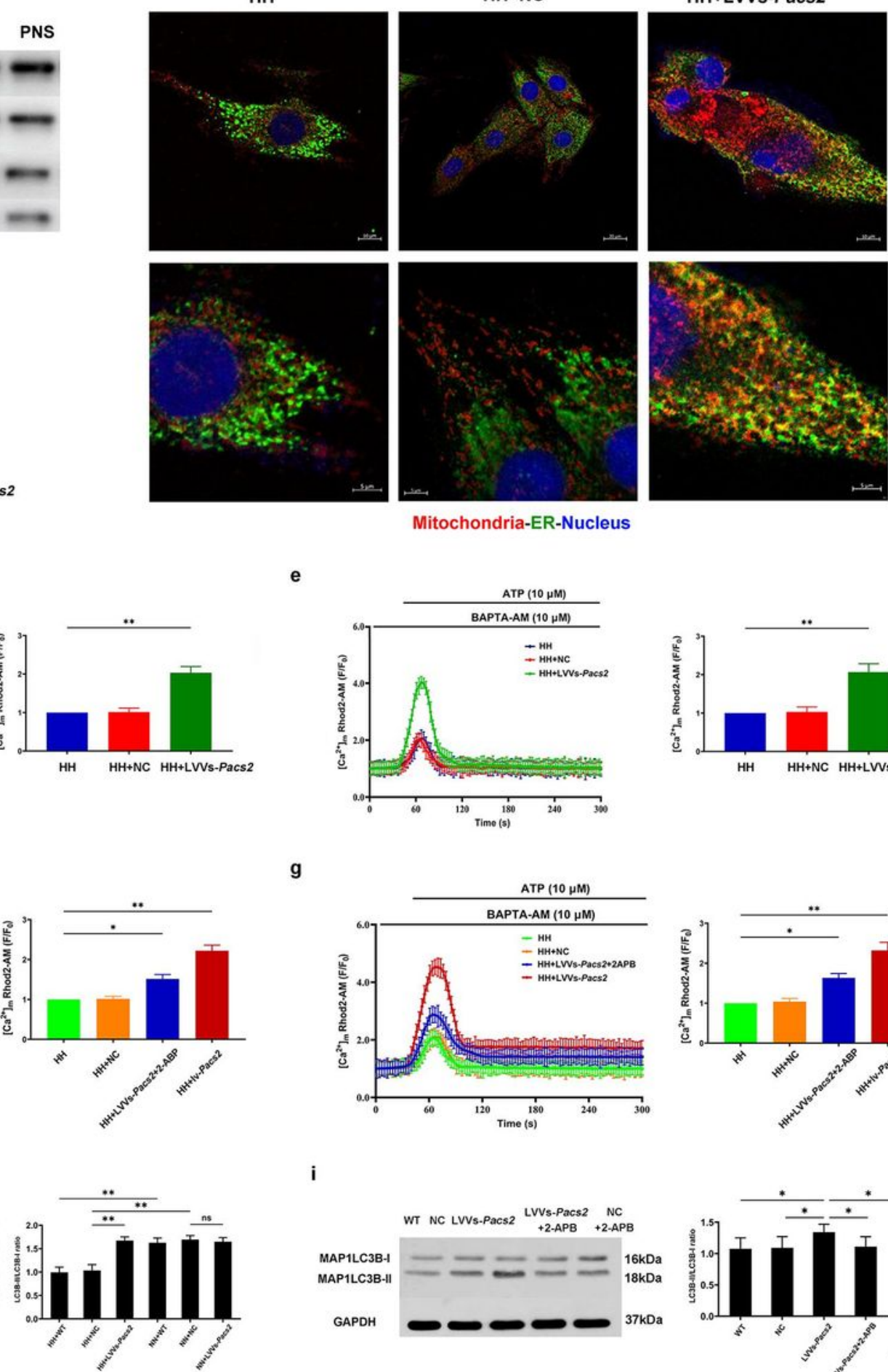

g
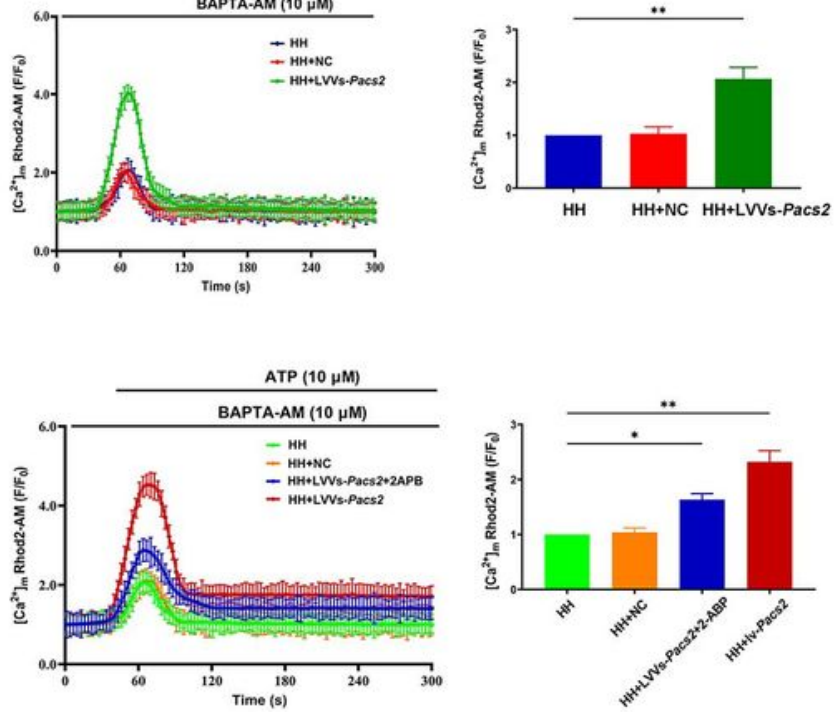

$\mathrm{HH} \quad \mathrm{HH}+\mathrm{NC} \mathrm{HH}+\mathrm{LWS}-\mathrm{Pacs} 2$
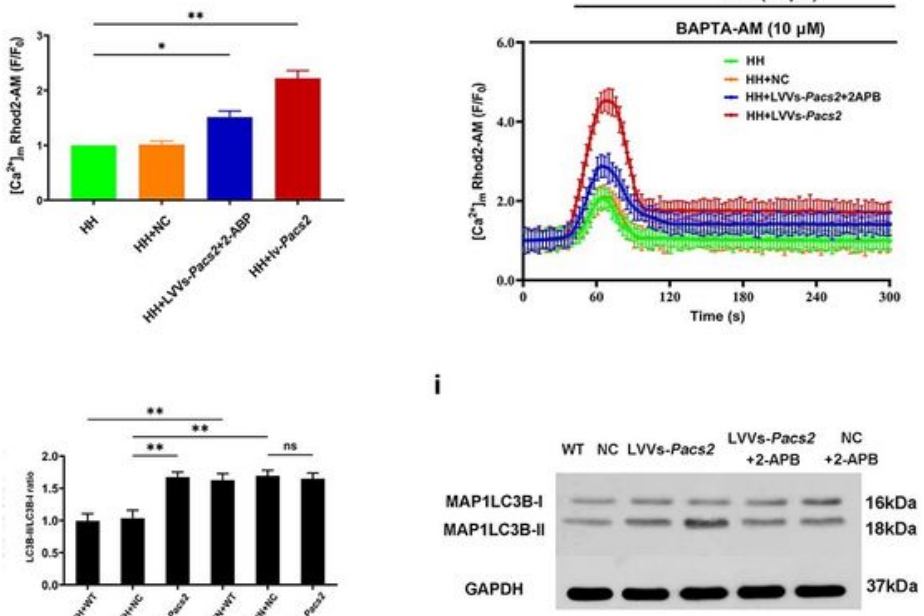

Figure 7

ER-mitochondria calcium flux is required for PACS2-mediated mitophagy. a) Western blot showed that Pacs2 is stably overexpressed in LVVs infected cardiomyocytes. b) Cardiomyocytes are coimmunostained for mitotracker (red) and ERP72 (green). Scale bar, $5 \mu \mathrm{m}$. c Pearson's overlap coefficient is employed to analyze the co-localization. $\mathbf{d}$, e) Calcium in the mitochondria marked by Rhod-2 AM indicating reversed TG (d) and ATP (e) mediated ER-mitochondria calcium fluorescence signals in Pacs2 
overexpression cells (green line) in HH condition (Cells without 2-APB treatment are used as controls). f, g) Mitochondria calcium evoked by LVVs overexpression of Pacs2 (red line) in the presence of TG (f) and ATP (g) were blocked by 2-APB (blue line). h) Western blots showing significantly increased MAP1LC3B-II turnover in LVVs-Pacs2 group. i) Western blots showing impaired PACS2-mediated MAP1LC3B-II turnover with the treatment of 2-APB. Cells are obtained from six mice per group, data are shown as mean $\pm \mathrm{SD}$, $P$ $<0.05$, $* \star P<0.01$. HH, simulated hypobaric hypoxia; NN, simulated normobaric normoxia; LVVs, Lentiviral vectors; ER, endoplasmic reticulum; TG, thapsigargin; ATP, adenosine triphosphate; 2-APB, 2aminoethoxydiphenyl borate.

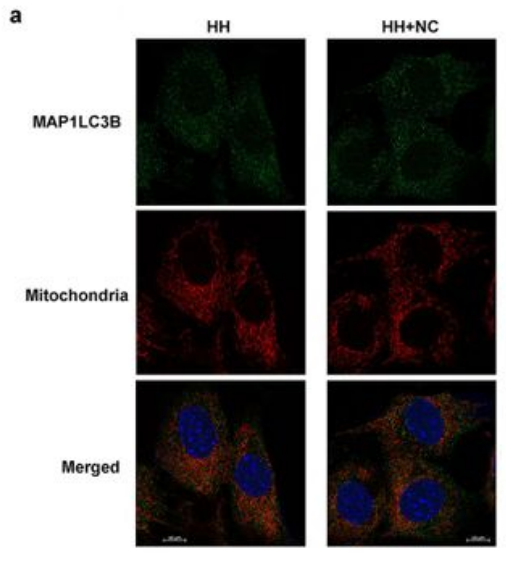

e
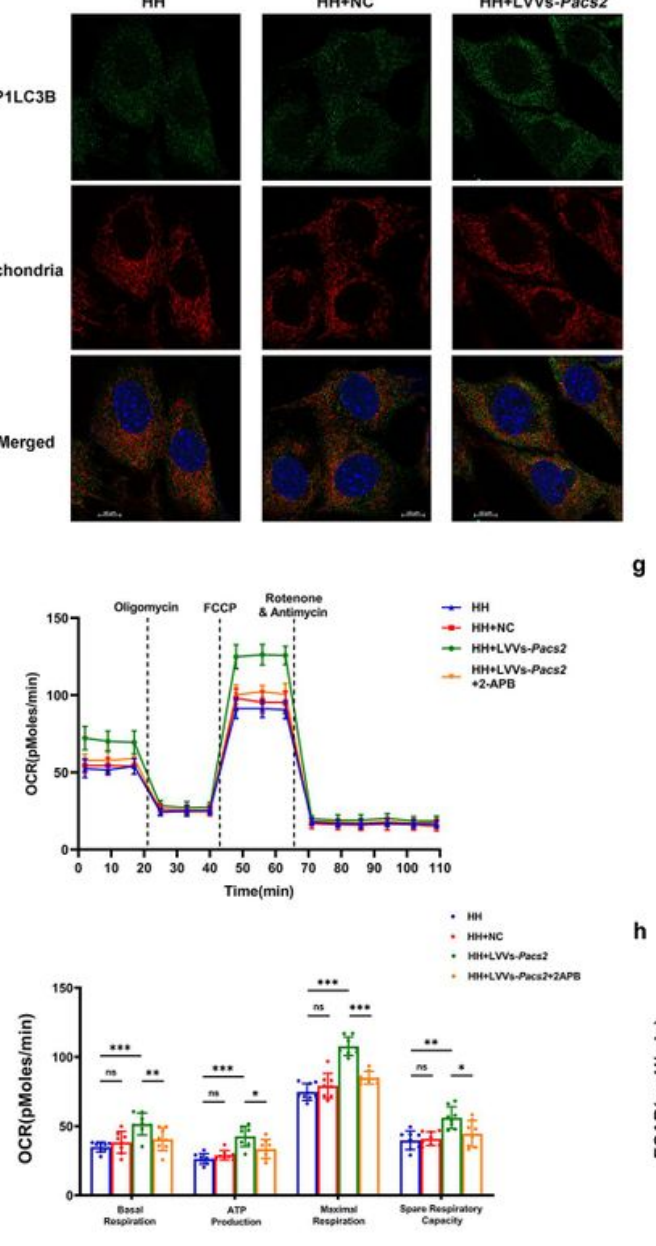

g
HH+LVVs-Pacs2 b
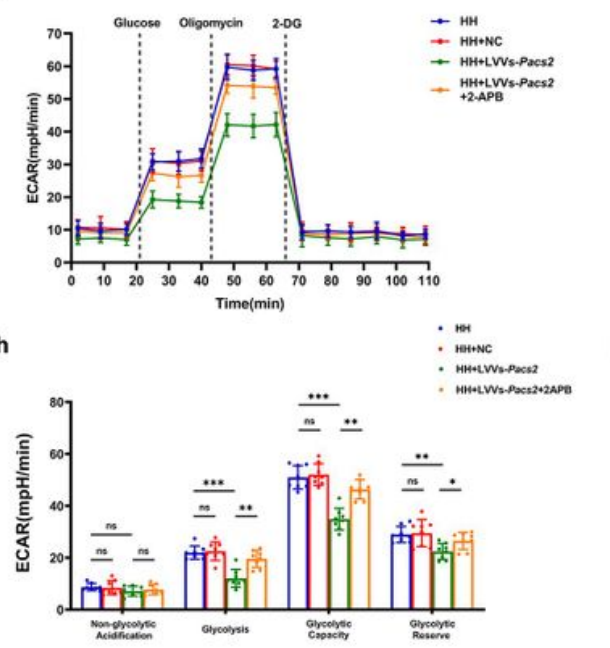

$\mathrm{HH}$

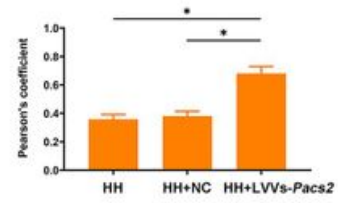

d
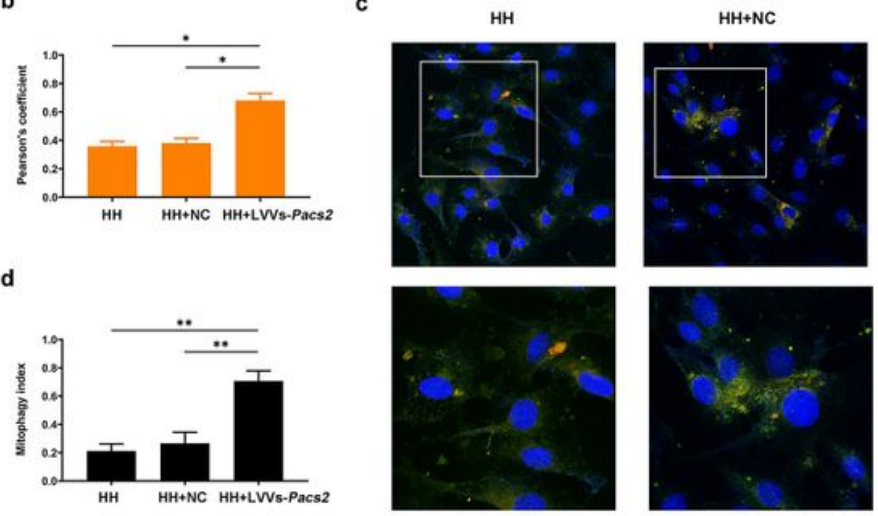

HH+LVVs-Pacs2
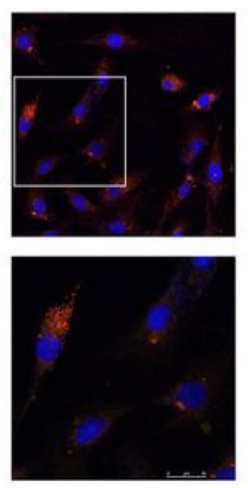

i
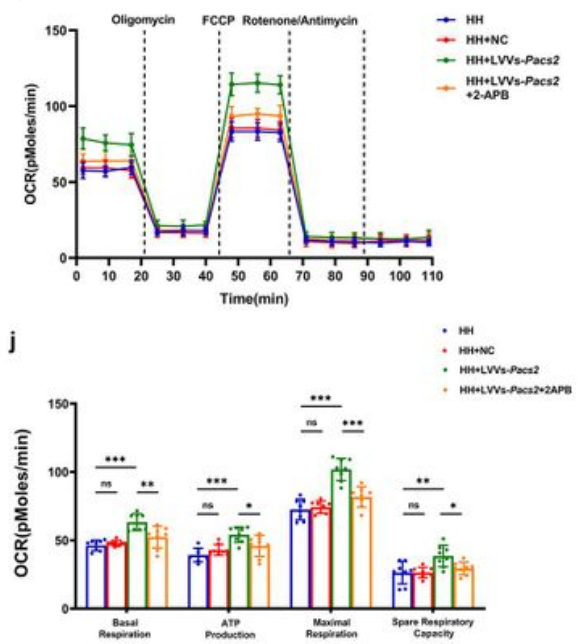

Figure 8

PACS2 supplementation alleviated impaired mitophagy and mitochondrial energy metabolism induced by hypobaric hypoxia. a, b) Mitotracker Deep Red and GFP-MAP1LC3B were used to mark H9C2 cardiomyocytes. Merged images (a) and Pearson's overlap coefficient analysis (b) revealing that the overexpression of Pacs2 increased the co-localization of MAP1LC3B-II and mitochondria during $\mathrm{HH}$ exposures. Scale bar, $10 \mu \mathrm{m}$. c, d) All three groups were infected by mtKeima plasmid for $12 \mathrm{~h}$ before HH exposure and observed under an LSCM. c) Representative images showing puncta formation in three groups. Scale bar, $25 \mu \mathrm{m}$. d) Quantitative analysis of the fluorescent area showing increased mitophagy 
index in LVVs-Pacs2 group. e) Measurement of the OCR of cardiomyocytes (blue), negative controls (red), LVVs-Pacs2 (green), and LVVs-Pacs2+2-APB (orange) in HH exposure. f) Basal respiration, ATP production, maximal respiration and spare respiratory capacity are calculated. $\mathbf{g}$ ) Measurement of the ECAR of cardiomyocytes (blue), negative controls (red), LVVs-Pacs2 (green), and LVVs-Pacs2+2-APB (orange) in $\mathrm{HH}$ exposure. h) Non-glycolytic acidification, glycolysis, glycolytic capacity and glycolytic reserve are calculated. i) OCR analysis of cells treated with BSA-conjugated palmitate substrate and after the sequential injection of each compound (oligomycin, FCCP, ETO) at the indicated concentration. j) Basal respiration, ATP production, maximal respiration and spare respiratory capacity are calculated with FAO substrate. $\mathrm{H} 9 \mathrm{C} 2$ cardiomyocytes are obtained from eight mice per group. Data are shown asmean \pm $\mathrm{SD},{ }^{*} P<0.05,{ }^{*} P<0.01$. HH, simulated hypobaric hypoxia; NN, simulated normobaric normoxia; laser scanning confocal microscopy; TG, thapsigargin; ATP, adenosine triphosphate; 2-APB, 2aminoethoxydiphenyl borate; OCR, oxygen consumption rate; ECAR, extracellular acidification rate; BAPTA-AM, bis-(aminophenolxy) ethane-N,N,N',N'-tetra-acetic acid acetoxyme-thylester; FCCP, trifluoromethoxy carbonyl cyanide phenylhydrazone; ETO, etomoxir.

a

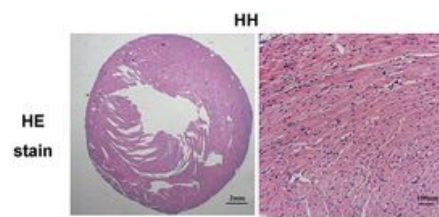

b

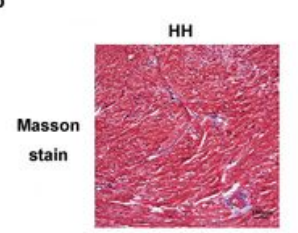

d
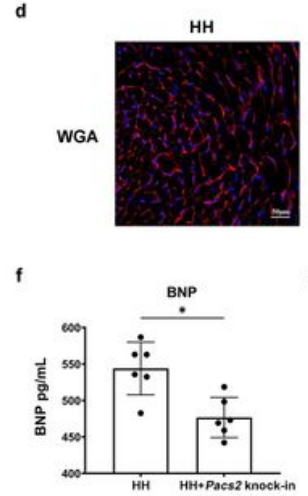

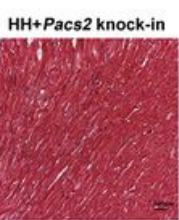

HH+Pacs2 knock-in
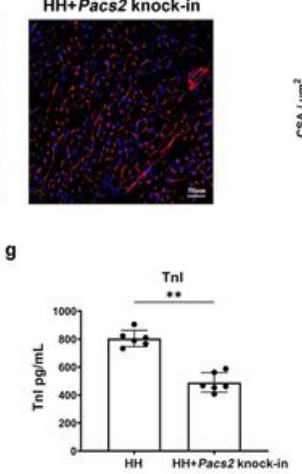

e
$\mathrm{HH}+$ Pacs2 knock-in

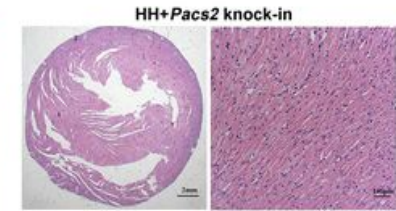

c
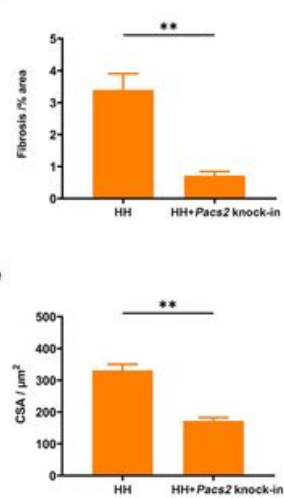

h

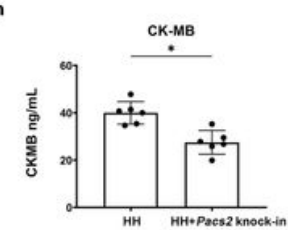

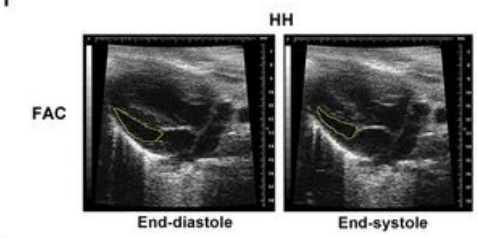

HH

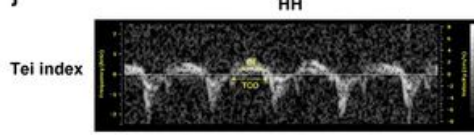

k
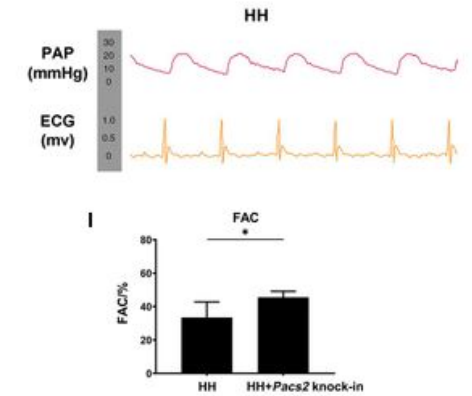

H+Pacs2 knock-in

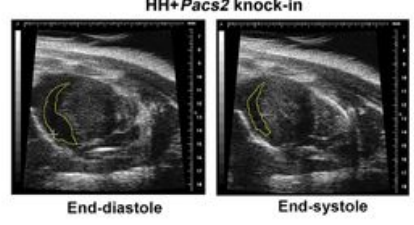

$\mathrm{HH}+$ Pacs2 knock-in
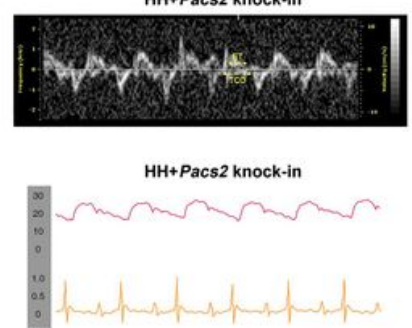

m

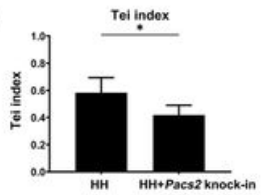

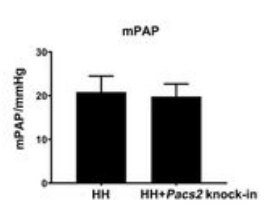

o

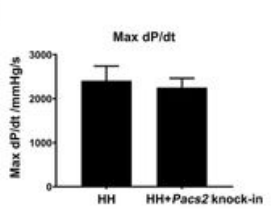

p

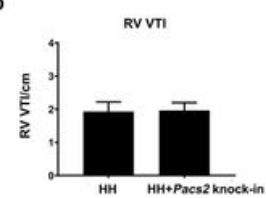

Figure 9

Cardiac Pacs2 knock-in alleviated hypobaric hypoxia-induced right myocardium injury and right cardiac dysfunction. a, b) Representative photographs of HE staining (a) and Masson's trichrome staining (b) of the right myocardium in cardiomyocyte-specific Pacs 2 knock-in heart and littermate controls. Scale bar, $100 \mu \mathrm{m}$. c) Quantification of fibrotic area revealing less myocardial fibrosis area (blue) in Pacs 2 knock-in mice. d) Representative images of right myocardium stained with WGA (red) to delineate sarcolemma 
and DAPI (blue). Scale bar, $50 \mu \mathrm{m}$. e) Bar graphs revealing cardiomyocytes CSA in the two groups. $\mathbf{f}-\mathbf{h}$ ) Statistics of plasma concentration of BNP, TNI and CK-MB of Pacs 2 knock-in mice and controls during $\mathrm{HH}$ exposure. i) FAC measurement of the RV in control group (FAC=28.51\%) and Pacs2 knock-in mice group ( $\mathrm{FAC}=50.68 \%$ ) in $\mathrm{HH}$ exposure. Representative images acquired at end-diastole (left) and endsystole (right). j) Tei index was measured in control group (Tei index $=0.57$ ) and Pacs2 knock-in mice group (Tei index=0.34). $\mathbf{k}$ ) mPAP measured by RHC and the ECG of control mice group (mPAP $=22.58$ $\mathrm{mmHg}$ ) and Pacs2 knock-in mice group (mPAP=21.67mmHg). I-p) Statistics of FAC (I), Tei index (m), $\operatorname{mPAP}(\mathbf{n}), \max \mathrm{dP} / \mathrm{dt}(\mathbf{0}), \mathrm{RV} V \mathrm{TI}(\mathbf{p})$ in the two groups. Right myocardium is obtained from six mice per group, data are shown asmean $\pm \mathrm{SD}, \star P<0.05,{ }^{\star} P<0.01$. HH, hypobaric hypoxia; NN, normobaric normoxia; RV, right ventricular; WGA, wheat germ agglutinin; CSA, cross-sectional area; BNP, brain natriuretic peptide; Tnl, troponin I; CK-MB, creatine kinase MB; RHC, right cardiac catheterization; FAC, fractional area change; mPAP, mean pulmonary artery pressure; VTI, velocity time integral. 

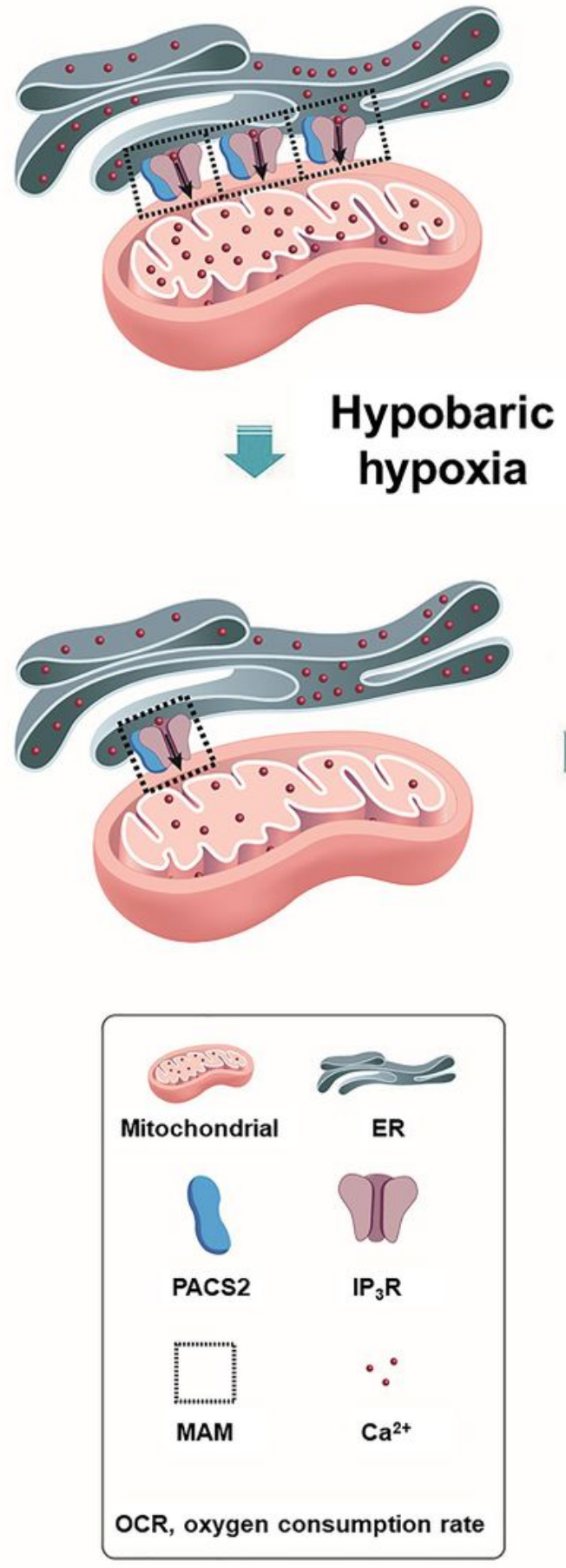

PACS2 $\downarrow$

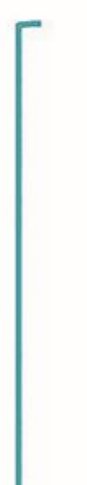

\section{Mitochondrial $\mathrm{Ca}^{2+}$ influx $\downarrow$}

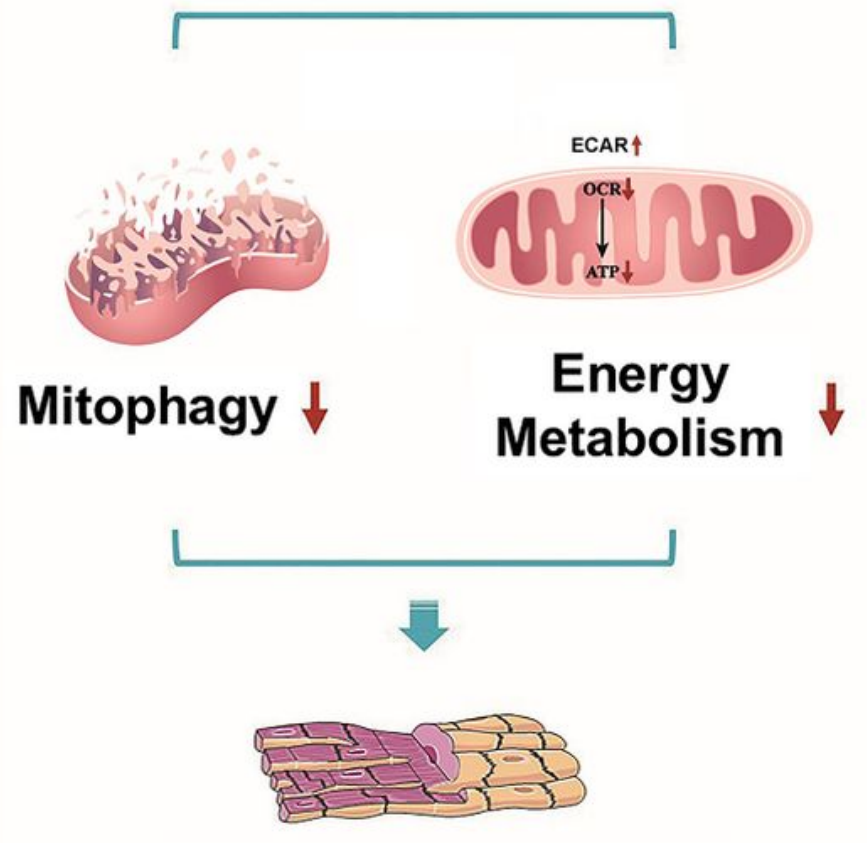

\section{Cardiomyocyte injury}

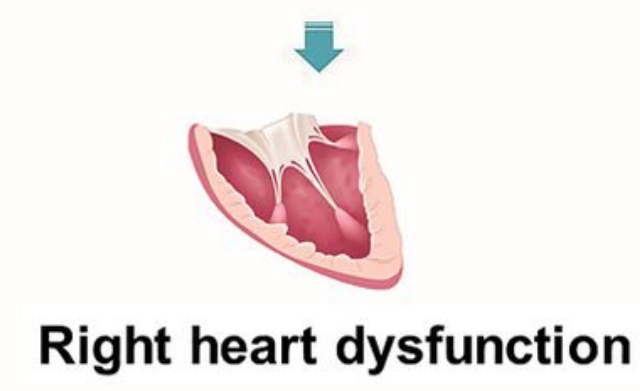

Figure 10

Graphic Abstract. A schematic drawing of the PACS2 modulated right cardiac dysfunction pathway under high-altitude exposure. $\mathrm{HH}$ is the major character at high altitude. Long-term $\mathrm{HH}$ reduces the expression of PACS2 in MAM. Insufficient PACS2 decreases MAM formation and ER-mitochondrial calcium flux, both of which resulted in mitophagy inhibition and mitochondrial energy metabolism impairment 


\section{Supplementary Files}

This is a list of supplementary files associated with this preprint. Click to download.

- SuppMetabolomics.xIsx

- SuppProteomics.xlsx

- SupplementalTable1.docx

- SupplementalTable2.doc

- Supplementarylnformation.pdf

- nrreportingsummary.pdf 